\title{
A Theory of Quantum Measurement Based on the CCR Algebra $\mathbf{L}^{+}(\mathscr{W})$
}

\author{
D. A. Dubin and J. Sotelo- $\dot{C} A$ MPOS $^{1.2}$ )
}

Ausgehend von der Observablen-Algebra $\mathbf{L}^{+}(\mathscr{W})$ für eịen Raum $\mathscr{W}$ vom Typ $\mathscr{S}$ wird eine quantenmechanische Meßtheorie ausgearbeitet. Diese geht auf die Theorie von Davies und Lewis zurück, wird aber hier für unbeschränkte symmetrische Operatoren mit einem gemeinsamen dichten Definitionsbereich und ohne Einschränkung an das Spektrum aufgebaut.

Исхоля из алгебры наблюдаемых $\mathbf{L}^{+}(\mathscr{W})$ для пространства $\mathscr{W}$ типа $\mathscr{S}$ разрабатывается ювантово-механическая теория измерения. Это теория восходит к Ден̆вис и Луис, однако адаптируется здесь к. неограниченным симметрическим операторам с общей плотной областью определения и без ограничения на спектр.

Starting from $\mathbf{L}^{+}(\mathscr{W})$ as the algebra of observables, $\mathscr{W}$ a space of type $\mathscr{I}$, a theory of quantum measurement is devised. It is based on the theory of Davies and Lewis, but adapted to unbounded symmetric operators defined on a common dense domain and no restriction on the spectra.

\section{Introduction}

The original von Neumann formulation of quantum measurement theory is based on two special circumstances: the pure states of the system constitute a Hilbert space, and the observables are self-adjoint operators with purely discrete spectra. Although von Neumann discussed the measurement of operators with continuous spectra, he based this on an approximation schene which is less than satisfactory.

We take the position that the states of the system must take finite values on all the observables, and the set of observables must include all the operators of physical interest, e.g., the position, momentum, and energy. In order that this be so, it turns out that the pure states of the system must comprise a topological vector space dense in the Hilbert space of the system. Following the work of RoBERTs [28; 29], Kristensen, Mejlbo, and Thoe-Poulsen [2] and others, we take the pure states to constitute a space $\mathscr{W}$ of type $\mathscr{S}$. The observables are taken to be the maximal *algebra of operators mapping $\mathscr{W}$ to itself; $\mathbf{L}^{+}(\mathscr{W})$. This means that we must develop a measurement theory for $\mathbf{L}^{+}(\mathscr{W})$.

- Davies and Lewis [23-25்] have developed a theory of measurements for bounded symnetric operators with general spectra. Using an approximation to the position operator, Davies has shown how this theory can be used for certain unbounded operators by constructing instruments for approximate position measurements [23]. For a connexion between these operational ideas and statistical decision theory, see the work of HoLevo [26, 27].

1) Open University Postgriduate Fellow.

2) Submitted in partial fulfillment of the requirements for the D. Phil. degree, The Open University. 
This leaves open the question of a theory along the lines of Davies and Lewis, but adapted to $\mathbf{L}^{+}(\mathscr{W})$. 'The problem is this. Instruments for measurements' must transform states on $\mathbf{L}^{+}(\mathscr{\mathscr { O }})$ to states. A further difficulty comes fróm the fact that $\mathbf{L}^{+}(\mathscr{W})$. is not complete in its natural topology. 'Its completion is the space of distributions $W^{\prime} \otimes W^{\prime}$. This places a further restriction on the definition of instrument: 'they must be the transpose of a map taking $\mathbf{L}_{+}^{+}(\mathscr{W})$ to itself. We call these latter maps expectations.

Each instrument defines a unique observable through a spectral representation by a positive operator-valued measure under which $\mathscr{W}$ is stable. The observable so defined serves as an approximation to certain other observables. The instrument in question then provides measurements for these associated observables, but providing less than maximum information. This phenomenon occurs in [24].

As is to be expected, strong repeatability is not generally possible. Somewhat sur-. prisingly, the composition of two instruments, corresponding to successive measurements, is not an instrument. This is not as bad as it seems, as the compose of two instruments behaves perfectly well on product sets $\Delta_{1} \times \Delta_{2}$ which could be taken to be all that is necessary,operationally.

The paper is organized as follows. In Section 2 we discuss the formalism of Quantum Mechanics. Here we introduce our space of wave functions, algebra of observables, and states. We also quote a number of results concerning this triple, as well as proving some new results we need for the sequel. In Section 3 we define expectations and Instruments in our model. We prove that instruments are bounded Radon measures in the sense of Tномas [39], that every instrument defines a unique observable, and we determine which observables can be measured. In Section 4 we consider composition and conditioning for instruments.'In Section 5 we construct a family of instruments to measure $Q$, and similar families for $P$ and $H$. We also prove that these instruments compose to instruments. In Section 6 we summarize our results through an informal discussion of the measuring, process.

The authors are pleased to acknowledge helpful conversations with P. M. CLARK, J. G. Clunie, G. I. Sewell, and especially with E. B. Davies. One of us, J. S.-C., gratefully acknowledges his appreciation to the Open University for its help and support, and the award of a postgraduate studentship. The other (D.A.D.) wishes to thank the members of the Naturwissenschaftlich-Theoretisches Zentrum of KarlMarx-Universität in Leipzig for their hospitality and discussions concerning this work. In particular, he wishes to thank G. LASSNER, G. A. LaSSNER, K. SCHMÜDgen, and A. Uhmann. He also wishes to thank C. Trapani for discussións clarifying the interpretation of this scheme.

\section{Formalism of quantum mechanies}

The essence of nonrelativistic quantum mechanics is the canonical commutation relations. If we demand that the space of pure states, $\mathscr{W}$, carries a representation of these relations, that the canonical operators be continuous linear operators on $\mathscr{W}$, then up to some technical conditions, $\mathscr{W}$ is determined.

Definition 2.1: The space $\mathscr{\mathscr { C }}[\mathrm{t}]$ of wave'functions for a system with $d$ degrees of freedom is the maximal locally convex space such that

(a) there exists a t-continuous scalar product, $\langle$,$\rangle on \mathscr{W}$. The completion of $\mathscr{W}$ with respect to this scalar product is a separable Hilbert space $\mathfrak{S}$; 
(b) there exist $d$ pairs $\left(b_{j}, b_{j}{ }^{*}\right)_{1 \geq j \geqq d}$ of continuous linear operators mapping $\mathscr{W}$ to itself, adjoint with respect to the scalar product

$$
\left\langle b_{j} f, g\right\rangle=\left\langle f, b_{j}^{*} g\right\rangle \quad\left(\forall f, g \in W_{;} ; 1 \leqq j \leqq \varliminf^{\prime} d\right), \quad .
$$

and satisfying the canonical commutation relations (CCR)

$$
\left[b_{j}, b_{k}^{*}\right]=\delta_{j \mathbf{k}} \quad(1 \leqq j, k \leqq d)
$$

strongly on $\mathscr{W}$, other commutators vanishing;

(c) the topology $t$ is determined by the seminorms

$$
f \rightarrow\|a f\| \quad\left(\forall a \in \mathbf{L}^{+}(\mathscr{W})\right),
$$

where

$$
\mathbf{L}^{+}(\mathscr{W})=\left\{\dot{a} \in \mathbf{L}(\mathscr{W}): a^{*} \in \mathbf{L}(\mathscr{W})\right\}
$$

and $\mathbf{L}(\mathscr{W})$ is the "set of all linear maps from $\mathscr{W}$ to itself. Here $\|\cdot\|$ is the norm associated with $\langle$,$\rangle ;$

(d) there is a vector $\Omega_{0} \in \mathscr{W}$, normalized by $\left\|\Omega_{0}\right\|=1$, satisfying the Fock-Cook condition

$$
b_{j} \Omega_{0}=0 \quad(1 \leqq j \leqq d) .
$$

(e) Let $\mathscr{W}_{v}$ be the lineàr manifold of all vectors in $\mathscr{W}$ satisfying the Fock-Cook condition. Then $\mathscr{P}_{\boldsymbol{v}}$ is dense in $\mathscr{W}$, where $\mathcal{P}$ is the algebra of all polynomials in the $\left\{b_{j}, b_{j}^{*}(1 \leqq j \leqq d)\right\}$. In addition, $\mathscr{W}$, is irreducible if $\mathscr{P} \Omega_{0}$ is dense in $\mathscr{W}$.

This choice of system was analyzed by Kristensen, Mejlbo, and Thue-Poursen [2], who called irreducible wave function spaces spaces of type $\mathscr{J}^{d}$, for reasons which will be immediately apparent. With regard to their analysis, note our condition of maximality and our choice of topology. Most of our results hold for more general spaces, but we, shall not elaborate on this possibility.

Proposition 2.2: (a) Every wave function space may be decomposed into the 1-completion of a countable locally convex direct sum of irreducible spaces:

$$
\mathscr{W}=\bar{\sum}_{n \geq 1}^{\oplus \mathscr{W}_{n}}{ }^{\mathrm{t}}
$$

(b) Any irreducible wave function space $\dddot{W}$ is tvs-isomorphic to Schwartz's space $\mathscr{S}^{d}=\mathscr{S}\left(\mathbf{R}^{d}\right)$ with its usual Frechet topology. Defining

$$
M=\sum_{1 \leqq j \leqq d} b_{j}^{*} b_{i}
$$

it follows that

$$
\mathscr{W}=\mathfrak{D}^{\infty}(M)=\bigcap_{p \geq 0} \operatorname{Dom}\left(M^{0}\right)
$$

The topology $\mathrm{t}$ is determined by the seminorms

$$
f \stackrel{\leftrightarrow \rightarrow}{\rightarrow}\|f\|_{p}=\left\|M^{p} f\right\| \quad(p \geqq 0) .
$$

Proof: The decomposition is effect by choosing an orthonormal basis $\left\{\Omega_{n}: n \geqq 1\right\}$ for the subspace $\{f: M f=0\}$. Define $\mathscr{W}_{n}$ to be the $t$-completion of $\mathscr{P} \Omega_{n}$, That $\mathscr{W}_{n}$ is an irreducible wave function. space is clear. For details and a proof that the $l c d s$ of the $\mathscr{W}_{n}$ is $\mathscr{W}$, see $[1: \S 4.4]$. The isomorphism of an irreducible $\mathscr{W}$ with $\mathscr{\mathscr { S }}$ is shown 
in $[1: \$ 4.10 ; 2]$. The identification of $\mathscr{S}^{d}$ with $\mathfrak{D}^{\infty}(M)$. is due to Srmon [3: V. 3 App.; 4], and is known as the $N$-representation. The topological result, that the usual topology on $\mathscr{S}^{d}$ is equivalent to the topology $t$, is a result of the closed graph theorem applied to $\mathbf{L}^{+}(\mathscr{W})$, in view of the fact that $t$ is the coarsest locally convex topology with respect to which every $a \in \mathbf{L}^{+}(\mathscr{W})$ is continuous

In what fóllows we shall abbreviate $\mathbf{L}^{+}(\mathscr{W})$ to $\mathcal{A}$, and write $a \rightarrow a^{+}$for the restriction of the $\mathfrak{W}$-adjoint to $\mathscr{W}$.

Let us remark that $t$ is generally known as the graph topology. This is because of the following inequalities: for all $a \in \mathcal{A}$ and all $f \in \mathscr{W}$,

$$
\|a f\| \leqq\|f\|+\|a f\| \leqq\left\|2^{-1 / 2}\left(a^{+} \dot{a}+1\right) f\right\| .
$$

Corollary 2.3: An irreducible wave function space, $\mathfrak{W}[\mathrm{t}]$, is nuclear and Frechet. Hence it is barreled, bornological, Mackey, Montel, reflexive, and separable. Its strong dual, consequently, is nuclear DF and complete, barreled, bornological, Mackey, Montel, reflexive, and separable. $W$ possesses an unconditional basis, the well known Hermite functions, $\left\{\Omega_{v}: \nu \in \mathbf{N}^{d}\right\}$. In Gel'fand's sense,

$$
\mathscr{W}[\mathrm{t}] \subset \mathfrak{Q} \subset \mathscr{W}^{\prime}\left[\mathrm{t}^{\prime}\right]
$$

constitutes a rigged triple.

Proof: The isomorphism $\mathscr{W} \approx \mathscr{S}^{d}$ implies that $\mathscr{W}$ is nuclear and Frechet. The. list of topological properties then'follows from. standard results in. tos theory, cf. [5: 33.2, 36.3, 56.14], [6: II.8.1, IV.5.7, IV.6.6], and [7: 4.3.3, 4.4.10, 4.4.12]. The Hermite functions are used to construct the trs isomorphism between $W$ and the sequence space $s^{d}$ : if $f=\sum c_{v} \Omega$, is an element of $\mathfrak{H}$, then $f \in \mathscr{W}$ if and only if $\left(c_{\nu}\right) \in s^{d}[1-4$, ibid. $]$

In equation (2.3) we introduced $\mathbf{L}^{+}(\mathscr{W})$, and, as noted below Prop. 2.2, we shall write

$$
\mathscr{c}=\mathbf{L}^{+}(\mathscr{W})
$$

For terminological purposes only; we shall refer to $A$ as our algebra of observables, and all clements of $\mathcal{A}$ as observables. There is no implication of physical measurability implied. Those elements of $\mathcal{A}$ which can be measured in our scheme, we, shall refer to as physical observables. Hereafter we shall assume $\mathscr{W}$ to be irredicible unless otherwise stated. The more general case then follows by countable direct sums.

Powers [8] has introduced a notion of self-adjointness for algebra of unbounded operators, and $\mathcal{A}$ satisfies the requirements.

Proposition 2.4: $\mathcal{A}$ is a complex unital *algebra' which is closed and solf-adjoint in the sense of Powers: respectively

$$
\mathscr{W}=\bigcap_{\mathfrak{A}} \operatorname{Dom}(\bar{a})=\bigcap_{\mathcal{A}} \operatorname{Dom}\left(a^{*}\right) .
$$

Proof: The second condition implies the first. The second condition follows because $\mathscr{W}[\mathrm{t}] \approx \dot{3}^{d}[9]$

In what follows we shall use the following more or less standard notation. if $E^{\prime}$ is an ordered vector space, $\mathbf{L}(E)$ is the set of all linear maps $E \rightarrow E$, and $\mathbf{L}_{+}(E)$ the subset of all positivity-preserving maps. If $E$ is a tvs as well, $\mathscr{L}(E)$ is the subset of $\mathbf{L}(E)$ consisting of all continuous maps on $E$, and $\mathscr{L}_{+}(E)$ is the subset of all positivitypreserving continuous maps. Similarly for $\mathbf{L}(E, F), \mathbf{L}_{+}(E, F), \mathscr{L}(E, F)$ and $\mathscr{L}_{+}\left(E^{i}, F^{\prime}\right)$. 
We shall have reasons to consider several topologies on $\mathcal{A}$. The tirst, which we introduce now, generalizes the uniform topology for bounded operators, and was first introduced by LASSNER $[10,11]$.

Proposition 2.5: Let $\mathfrak{u}$ be the topology of uniformly bounded convergence induced on $\mathcal{A}$ from $\mathscr{L}\left(\mathscr{W}[\mathrm{t}], \mathscr{W}^{\prime}\left[\mathrm{t}^{\prime}\right]\right)$, i.e., generated by the seminorms $(\forall$ bounded $\mathscr{M} \subset \mathscr{W}[\mathrm{t}])$

$$
\|a\|_{(\mathscr{N})}=\sup \{|\langle a f, g\rangle|: f, g \in \mathscr{M}\} \text {. }
$$

Equipped with this topology, $\mathcal{A}$ is a topological *algebra, i.e., the involution is continuous and the product is separately continuous, as well as $\mathcal{A}[\mathrm{u}]$ being a topological - vector space.

As a tvs, $\mathcal{A}[u]$ is incomplete. Its completion is $\mathscr{W}^{\prime}\left[\mathrm{t}^{\prime}\right] \otimes \mathscr{W}^{\prime}\left[\mathrm{t}^{\prime}\right]$, the completed projective tensor product. Hence $\mathrm{u}$ is nuclear.

The topology $\mathfrak{u}$ is determined through $M$ by means of the equivalent family of seminorms

$$
\|a\|_{\varphi}=\|\| \varphi(M) a \varphi(M)\|\|
$$

where \|\|$\cdot \|$ is the operator norm on $\mathbf{B}(\mathfrak{S})$, and $\phi$ runs through the space

$$
\left\{\varphi: \mathbf{R}^{+} \rightarrow \mathbf{R}^{+}: \varphi_{1} \text { is bounded, continuous, and } \sup _{x}\left|x^{k} \varphi(x)\right|<\infty(k \geqq 0)\right\} \text {. }
$$

The following subsets of $\mathscr{A}$ are important in the sequel.

Definition 2.6: An element $a \in \mathcal{A}$ is said to be symmetric, or hermitian, if $a^{+}=a$. The set of all hermitian elements of $\mathcal{A}$ is denoted by $\mathcal{A}_{\mathrm{h}}$. An element $a \in \mathcal{A}_{\mathrm{h}}$-is said to be positive if, for all $f \in \mathscr{W}$,

$$
\langle a \hat{f}, f\rangle \geqq 0 \text {, }
$$

and then we write $a \geqq 0$. The set of all positive elements in $\mathcal{A}_{\mathrm{h}}$ is denoted by $\mathscr{A}_{+}$.

Proposition 2.7: (a) $\mathcal{A}_{\mathrm{h}}$ is a real vector subspace of. $\mathcal{A}$.

(b) $\mathcal{A}_{+}$is a proper cone and determines a partial order in $\mathcal{A}_{n}$, with respect to which $\mathcal{A}_{\mathrm{h}}$ is an ordered vector space: $a \geqq b$ iff $a-b \in \mathcal{A}_{+}$, and $a=b$ iff $a \geqq b$ and $b \geqq a$.

(c) $\mathcal{A}_{+}$is a normal cone in $\mathcal{A}_{\mathrm{h}}$ which is generating.

(d) The order topology on $\mathcal{A}$, $\varrho$, is given explicitly through the seminorms

$$
\varrho_{x}(\bar{a})=\sup \left\{|\langle a f, f\rangle|\langle\langle x f, f\rangle: f \in \mathscr{W}\} \quad\left(x \in \mathcal{A}_{+}\right),\right.
$$

where $c / 0^{-}=+\infty$ and $0 / 0=0, c \in \mathbf{R}^{+}$. Let $\mathscr{N}_{x} \subset \mathcal{A}$ be the subset on which $\varrho_{x}$ is finite; $\varrho$ is the inductive limit topology:

$$
\mathcal{A}[\underline{g}]=\lim _{x \in \mathcal{A}_{+}} \text {ind } \mathscr{N}_{x}\left[\varrho_{x}\right]:
$$

Then $\varrho=\mathrm{u}$ and so $\mathcal{A}[\mathrm{u}]$ is bornological.

Proof: (a) Set $a=a_{1}+i a_{2}$, with $a_{1}=\left(a+a^{+}\right) / 2$ and $i a_{2}=\left(a-a^{+}\right) / 2$. (b) Clearly $\mathcal{A}_{+}$is a wedge. If $a,-a \in \mathcal{A}_{+}$; then $\langle a f, f\rangle=0$ for all $f \in \mathscr{W}$. Choosing . $f=\alpha f_{1-}+\beta f_{2}$ leads to $\langle a f, g\rangle=0$ for all $f, g \in \mathscr{O}$; hence $a=0$, and $\mathcal{A}_{+}$is a cone. (c) For all $a \in \mathcal{A}_{\mathrm{h}}$, set $a=a_{1}-a_{2}$, with $4 a_{1}=(1+a)^{2}$ and $4 a_{2}=(1-a)^{2}$, so that $\mathcal{A}_{+}$is generating: $\mathcal{A}_{\mathrm{n}}=\mathcal{A}_{+}-\mathcal{A}_{+}$. For normality, see [12:4.1]. (d) The $\varrho$-topo$\log y$ was introduced in [13]. Since each $\varrho_{x}$ is an order unit norm, $\varrho$ is the order topology [6]. That $\varrho=u$ was shown by SchmöDGEN [14:'Cor. 2 to Th. 1]

Now states are positive functionals (c.f. below) and the collapse of a wave packet, being a-map from states to states, requires the notion of a positivity-preserving map. 
Proposition 2.8: Every positive linear map $\dot{F} \in \mathbf{L}_{+}(\mathcal{A}[\mathfrak{H}])$ is continuous and

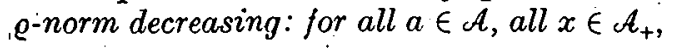

$\cdot \varrho_{F(x)}[F(a)] \leqq \varrho_{x}(a)$

Hence $\mathbf{L}_{+}(\mathscr{A})=\mathscr{L}_{+}(\mathcal{A})$.

\section{For a proof, see [13]}

From an abstract point of view, the set $\left\{\sum_{1 \leq j \leqq n} a_{j}{ }^{+} a_{j}: a_{j} \in \mathcal{A}, n \geqq 1\right\}$ is a more natural. cone for $\mathcal{A}$ than is $\mathscr{A}_{+}$. For applications to quantum measurement theory, however, only $\mathcal{A}_{+}$is needed; hence we have an unambiguous use of the term positive. In the mathematical literature, what we call positive is rightly called strongly positive, as the above cone is always a subset of $\mathscr{A}_{+}$.

Let us consider next the dual of $\mathcal{A}$ and its structure. We start with some defi, nitions.

Definition 2.9: (a) The dual of $\mathscr{A}[u]$, written $\mathscr{A}^{\prime}$, is the set of all continuous linear functionals $\varphi: \mathcal{A} \rightarrow$ C. A functional $\varphi$ is said to be hermitian if, for all $a \in \mathcal{A}$,

$$
\overline{\varphi(a)}=\varphi\left(a^{+}\right)
$$

the set of all hermitian functionals is denoted by $\mathcal{A}_{\mathrm{h}}{ }^{\prime}$. A functional $\varphi$ is said to be positive if, for all $a \in \mathcal{A}_{+}$;',

$$
\varphi(a) \geqq 0 \text {; }
$$

the set of all positive functionals is denoted by $\mathcal{A}_{+}{ }^{\prime}$. A state $\varphi$ is a positive functional which is normalized by

$$
\varphi(1)=1 \text {; }
$$

the set of states is denoted by $\mathbf{E}(\mathcal{A})$, or simply $\mathbf{E}$.

(b) The following subsets of $\mathbf{B}(\mathfrak{W})$ are important.

$$
\Im(\mathcal{A})=\left\{t \in \mathbf{B}(\mathfrak{Q}): \text { for all } a \in \mathcal{A}, t a, t^{*} a \text { are nuclear }\right\}
$$

the set $\Im(\mathcal{A})_{\mathrm{h}}$ of self-adjoint elements of $\Im(\mathscr{A})$, and the set $\Im(\mathcal{A})_{+}$of positive elements of $\Im(\mathscr{A})$. For brevity we often write $\Im$, $\mathfrak{\Im}_{\mathrm{h}}$, and $\Im_{+}$.

The next proposition makes precise the statement that all states are "density matrices".

Proposition 2.10: (a) $\Im=\Im_{\mathrm{n}}+i \Im_{\mathrm{h}}$ and $\mathfrak{\Im}_{+}$is generating for $\mathfrak{\mho}_{\mathrm{h}}: \mathfrak{\Im}_{+}-\Im_{+}=\mathfrak{\Im}_{\mathrm{n}}$. We have, further, that

$$
t \in \mathfrak{Y} \text { implies } t, t^{*}: \mathfrak{D} \rightarrow \mathscr{W} \text {. }
$$

(b) A linear functional on $A$ is continuous if and only if it is of the form

$$
\varphi(a)=\operatorname{tr}(t a) \quad(\forall a \in \mathcal{A})
$$

for some $t \in \Im$. Such functionals are said to be normal. Moreover, $\varphi \in \mathcal{A}_{+}$' if and only if $t \in \Im_{+}$. Hence, the trace determines an isomorphism between $\mathcal{A}_{+}{ }^{\prime}$ and $\Im_{+}, \mathcal{A}_{\mathrm{h}}{ }^{\prime}$ and $\Im_{\mathrm{h}}$, and $\mathcal{A}^{\prime}$ and $\Im$.

(c) If $\varphi \in \mathbf{E}$, and $\varphi(a)=\operatorname{tr}(t a)$, then

$$
\operatorname{tr}(t)=1 \text {, }
$$

and $t$ is said to be a density matrix. 
(d) The trace is cyclic: for all $a \in \mathcal{A}, t \in \Im$;

$$
\operatorname{tr}(t a)=\operatorname{tr}(a t) \text {. }
$$

For a proof see $[15-19]$

In [15] SCBMCÜDGEN points out that the character $\delta_{x}: f_{\rightarrow} \rightarrow f(x)$ is a positive functional on $\mathbf{B}\left[L^{2}(0,1)\right]$ which is not a trace: not all states on $\mathbf{B}(\mathfrak{W})$, are normal.

Proposition 2.11: The extreme states of the convex set $\mathbf{E}$ are of the form

$$
\varphi(a)=\operatorname{tr}(P a), \quad(a \in \mathcal{A})
$$

where $P_{f}$ is the orthogonal projection onto the vector $f \in \mathscr{W}$ :

$$
P_{r}(g)=\langle g, f\rangle f . \quad(g \in \mathscr{W}) .
$$

Hence, the points of $\mathscr{W}$ are in one-to-one correspondence with the pure states.

Proof: Let $\varphi \in \mathcal{A}_{+}^{\prime}$ and $\varphi=\varphi_{1}+\varphi_{2}$ with corresponding density operators $\varrho, \varrho_{1}, \varrho_{2}$; Define $\sigma=\varrho-\dot{\varrho}_{1}-\varrho_{2}$; so that $\sigma$ is trace class and $\operatorname{tr}(\sigma a) \doteq 0$, all $a \in \mathcal{A}$. Considering all rank one operators in gives $\langle\sigma f, g\rangle=0$, for all $f, g \in \mathscr{W}$; and so $\sigma=0$.

Suppose now that $\varrho$ is the projection on' the subspace spanned by $f \in \mathscr{W}$. Then $\varrho$ vanishes on the orthogonal complement; by positivity, so do $\varrho_{1}$ and $\varrho_{2}$, whence $\varrho$ is extreme. Conversely, if $\varrho$ is not a one dimensional projection, as a density matrix it can be decomposed into two or more projections, and is not extreme

Hereafter we shall identify $\Im$ with $\mathcal{A}^{\prime}$ : For notational purposes, if $\varphi \in \mathscr{A}^{\prime}$ is a linear functional, we shall write $\varphi(a)=\operatorname{tr}(\hat{\varphi} a)$, and so $\hat{\varphi} \in \mathfrak{Z} \subset \mathbf{B}(\mathfrak{Y})$ as above.

Our measurement theory requires an analysis of the topological structure of $\left(\mathcal{A}, \mathcal{A}^{\prime}\right)$ as a dual pair. We start by proving duality, and then we introduce a number of important topologies for the pair.

Lem ma 2,12: $\left[\mathcal{A}, \mathscr{A}^{\prime},\langle;\rangle\right]$ is a dual pair, where

$$
\langle a ; t\rangle=\operatorname{tr}(t a)
$$

That is to say,

$$
\begin{array}{ll}
\langle a ; t\rangle=0 & \text { for all } t \in \mathcal{A}^{\prime} \text { implies } a=0 ; \\
\langle a ; t\rangle=0 & \text { for all } a \in \mathcal{A} \text { implies } t=0
\end{array}
$$

Proof: We only prove the first condition. Let $\langle a ; t\rangle=0$ for all $t$, in particular for $t_{g}(f)=\langle f, a g\rangle g$, äll $g \in \mathscr{W}$. Then $\left\langle a ; t_{g}\right\rangle=\|a g\|^{2}$, and so $a=0$.

Definition 2.13: The coarsest locally convex topology on $\mathcal{A}$ 'compatible with the above duality is denoted $\sigma\left(\mathcal{A}, \mathcal{A}^{\prime}\right)=\sigma$; similarly for $\sigma\left(\mathcal{A}^{\prime}, \mathcal{A}\right)=\sigma^{*}$ on $\mathcal{A}^{\prime}$.

The strong topologies with respect to the above duality are denoted by $\beta\left(\mathcal{A}, \mathcal{A}^{\prime}\right)=\beta$ and $\beta\left(\mathcal{A}^{\prime}, \mathcal{A}\right)=\beta^{*}$, on $\mathcal{A}$ and $\mathscr{A}^{\prime}$ respectively. The strong topologies are defined by. the seminormis

$$
\begin{array}{ll}
\beta: a \nrightarrow \sup \{\mid \varphi(a) !: \varphi \in \mathscr{N}\}, & \text { all weakly bounded } \mathscr{N} \subset \mathcal{A}^{\prime}, \\
\beta^{*}: \dot{\varphi} \rightarrow \sup \{|\varphi(a)|: a \subset \mathcal{A}\}, & \text { all weakly bounded } \mathcal{M} \subset \mathcal{A} .
\end{array}
$$

The finest locally convex topologies on $\mathcal{A}, \mathscr{A}^{\prime}$ compatible with the duality are the Mackey topologies $\tau\left(\mathcal{A}, \mathcal{A}^{\prime}\right)=\tau$ and $\tau\left(\mathcal{A}^{\prime}, \mathcal{A}\right)=\tau^{*}$ respectively.

The structure of $\mathscr{A}^{\prime}\left[\beta^{*}\right]$ will be useful in our theory of measurement. The following is the pertinent result. 
Proposition 2.14: (a) For the above-defined topologies,

$$
\begin{array}{ll}
\sigma \leqq u \leqq \tau \leqq \beta & \text { on } \mathcal{A} \\
\sigma^{*} \leqq \tau^{*}=\beta & \text { on } \mathcal{A}^{\prime} .
\end{array}
$$

Hence $\beta$ is not generally compatible with the duality, although $\beta^{*}$ is.

(b) The following tvs isomorphisms hold:

$$
\mathcal{L}^{\prime}\left[\beta^{*}\right] \approx \mathscr{W}[\mathrm{t}] \otimes \mathscr{\mathscr { C }}[\mathrm{t}] \approx \mathscr{S}\left(\mathbf{R}^{2 d}\right),
$$

the last with its usual topology. The $\beta^{*}$-topology may be described by the norms

$$
\||\varphi| !\|_{n}=\left\|\mid M^{n} \hat{p} M^{n}\right\| ! \quad(n \geqq 0) ;
$$

where $\varphi(a)=\operatorname{tr}(\hat{\varphi} a)$ and $\|\cdot \mid\|$ is the operator norm on $\mathbf{B}(\tilde{\mathfrak{Q}})$. Hence $\mathcal{A}^{\prime}\left[\beta^{*}\right]$ is a nuclear Frechet lmc *algebra. It is barreled, bornological, complete, Mackey $\left(\beta^{*}==\tau^{*}\right)$, Montel, reflexive, and separable.

(c) The real subspace $\mathcal{L}_{\mathrm{h}}{ }^{\prime}\left[\beta_{\mathrm{h}}{ }^{*}\right]$ is nuclear and Frechet, and so enjoys the above topological properties. Here $\beta_{\mathrm{h}}{ }^{*}=\beta^{*}+A \mathcal{A}_{\mathrm{h}}{ }^{\prime}$.

(d) Let $F \in \mathbf{l}_{+}(\mathcal{A})$; then $F$ is u-continuous, and its transpose $F^{t} \in \mathscr{L}_{+}\left(\mathcal{A}^{\prime}\left[\beta^{*}\right]\right)$ exists and is $\beta^{*}$-continuous.

(e) Let $G^{\prime} \in \mathbf{L}_{+}\left(\mathcal{A}_{\mathrm{h}}{ }^{\prime}\right)$; then $G$ is $\beta_{\mathrm{h}}{ }^{*}$-continuous.

(f) The cone $\mathcal{A}_{+}{ }^{\prime}$ is closed and normal in $\mathcal{A}_{\mathrm{h}}{ }^{\prime}\left[\beta_{\mathrm{h}}{ }^{*}\right]$, and has empty interior.

Proof: (a) Everything is immediate from the définitions, save $\beta^{*}=\tau^{*}$ which we shall prove below: (b) See $[19,21]$. (c) We need only prove $\mathcal{A}_{\mathrm{n}}{ }^{\prime}$ Frechet, as nuclearity is clear. As $\Im\left[\beta^{*}\right]$ is complete and the involution is continuous, $\mathcal{A}_{\mathrm{h}}{ }^{\prime}$ is a closed subspace, hence Frechet: (d) The first part is Prop. 2.8. Thus $F$ is $\sigma$-continuous, implying that $F^{t}$ exists and is $\sigma^{*}$-continuous [6: IV.2.1], and is obviously positive. From [6: IV.2.4] it follows that $F^{t}$ is $\beta^{*}$-continuous. (e) As $\mathcal{A}_{\mathrm{h}}{ }^{\prime}\left[\beta_{\mathrm{h}}{ }^{*}\right]$ is Frechet and Mackey, and $\mathscr{A}_{+}^{\prime}$ is generating, then every positive linear form is continuous [6]; consequently, every linear map in $\mathbf{L}_{+}\left(\mathcal{A}_{\mathrm{h}}{ }^{\prime}\right)$ is continuous [6]. (f) Let $\left(\varrho_{\alpha}\right)$ be a net in $\mathcal{A}_{+}{ }^{\prime}$ converging to $\varrho \in \mathcal{A}_{\mathrm{b}}$ ' in the $\beta_{\mathrm{h}}{ }^{*}$-topology. Hence it is norm-convergent, whence $\varrho$ is positive, so $\varrho \in \mathcal{A}_{+}^{\prime}$, and so $\mathcal{A}_{+}^{\prime}$ is closed. We show that $\mathcal{A}_{+}^{\prime}$ is normal by using the norms $(2.37)$. For all $\varphi, \psi \in \mathcal{A}_{+}^{\prime}$ and all indices $n \geqq 0$,

$$
\begin{aligned}
\left\|\varphi+\psi^{\prime}\right\| \|_{n}=\sup \left\{\left\langle(\hat{\varphi}+\hat{\psi}) M^{n} f, M^{n} f\right\rangle: f \in \mathscr{W},\|f\|=1\right\} \\
\geqq \sup \left\{\left\langle\hat{\varphi} M^{n} f ; M^{n} f\right\rangle: f \in \mathscr{W},\|f\|=1\right\}=\|i \mid \varphi\|_{n}^{-} .
\end{aligned}
$$

As $\mathcal{A}_{\mathrm{h}}{ }^{\prime}\left[\beta_{\mathrm{h}}{ }^{*}\right]$ is non-normable and $\mathcal{A}_{+}{ }^{\prime}$ is normal, $\mathcal{A}_{+}{ }^{\prime}$ has no interior points $[6: \operatorname{Ex} .10(\mathrm{c})$, p. 252]

For later purposes, we need the following technical results concerning the order properties of $\iota^{\prime}$.

Lenma 2.15: Let $\left(\varphi_{n}\right)_{\mathrm{N}}$ be a monotonically increasing sequence of hermitian functionals $\varphi_{n} \in \mathcal{A}_{\mathrm{h}}{ }^{\prime \prime}$, such that for all $p=0,1,2, \ldots$,

$$
\lim _{n} \varphi_{n}\left(M^{2 p}\right)<\infty \text {. }
$$

Then there exists a unique $\varphi \in \mathcal{A}_{\mathrm{h}}{ }^{\prime}$ to which the sequence converges in the $\beta_{\mathrm{h}}{ }^{*}$-topology:

$$
\beta_{\mathrm{h}}{ }^{*} \cdot \lim _{n} \varphi_{n}=\varphi \text {. }
$$

Proof: Because of $(2.39 . \mathrm{a}),\left\{\left|\varphi_{n}\left(M^{2 p}\right)\right| \vdots n \geqq 1\right\}$ is a real Cauchy sequence for each $p \geqq 1$. Introducing the signum function

$$
(, m)=\left\{\begin{array}{l}
+1 \text { if } n \geqq m, \\
-1 \text { if } \cdot n<m,
\end{array} .\right.
$$


it follows that for all $n, m \geqq 1$,

$$
s(n, m)\left[\varphi_{n}-\dot{\varphi}_{m}\right] \in \mathcal{A}_{+}^{\prime} \quad \text { and } s(n, m)\left[\varphi_{n}-\dot{\varphi}_{m}\right](a)=\left|\left[\varphi_{n}-\varphi_{m}\right](a)\right| \text {. }
$$

We show that $\left(\varphi_{n}\right)_{\mathrm{N}}$ is a $\beta_{\mathrm{h}}{ }^{*}$-Cauchy sequence:

$$
\begin{aligned}
\|\| \varphi_{n}-\varphi_{m}\|\|_{p} & =\|\| s(n, m)\left[\varphi_{n}-\varphi_{m}\right] \mid \|_{p} \\
& \leqq \operatorname{tr}\left(M^{p} s(n, m)\left[\hat{\varphi}_{n}-\hat{\varphi}_{m}\right] M^{p}\right\rangle=\left|\varphi_{n}\left(M^{2 p}\right)-\varphi_{m}\left(M^{2 p}\right)\right| .
\end{aligned}
$$

The result now follows from the completeness. of $\beta_{\mathrm{h}}{ }^{*}$

Corollary 2.16: Let $\left(\varphi_{n}\right)_{N}$ be an upper bounded, monotonically increasing sequence in $\mathcal{A}_{\mathrm{h}}{ }^{\prime}$. Then there exists a $\varphi \in \mathcal{A}_{\mathrm{h}}{ }^{\prime}$ such that $\beta_{\mathrm{h}}{ }^{*} \cdot \lim _{n} \varphi_{n}=\varphi ;$ moreover $\varphi \doteq \sup \left\{\varphi_{n}: n \geqq 1\right\}$.

Using this result and [6: Cor. 2, p. 224] yields the following.

-Proposition 2.17: $\mathcal{A}_{\mathrm{h}}^{\prime}$ is monotone complete, and $\beta_{\mathrm{h}}{ }^{*}$ is compatible with the $\mathcal{A}_{+}$'-partial order. Hence $\mathcal{A}_{\mathrm{h}}{ }^{\prime}$ is monotone $\sigma$-complete.

Recall that if $\varphi \in \mathbf{N}$ is a state, the spectral theorem asserts the existence of an orthonormal basis for $\mathfrak{Q},\left\{e_{n} \in \mathscr{W}: n \geqq \cdot 1\right\}$, and a sequence $\left\{t_{n} \geqq 0: n \geqq 1\right\}$ of positive reals with $\sum_{n} t_{n}=1$, such that

$$
\lim _{n \rightarrow \infty} \operatorname{tr}\left[\hat{\varphi}-\sum_{1 \leqq j \leqq n} t_{j} P_{i}\right]=0 .
$$

Here $P_{j}$ is the orthogonal projection onto $e_{n}$. The relation between this expansion and the $\beta^{*}$-topology is this.

Proposition 2.18: Using the notation above,

$$
\beta^{*} \cdot \lim _{n}\left[\hat{\varphi}-\sum_{1 \leqq j \leqq n} t_{j} P_{j}\right]=0 \text {. }
$$
Proof: Ict us abbreviate $\hat{\phi}-\sum_{1 \leqq j \leqq n} t_{j} P_{j}=\hat{\phi}_{n}$. Now $\hat{\varphi}_{n} \geqq 0$ and for all $p \geqq 0$,
$M^{p} \hat{\phi}_{n} M^{p}$ is nuclear. Hence

$$
\left\|\mid \hat{\varphi}_{n}\right\|\left\|_{p} \leqq \operatorname{tr}\left(M^{p} \hat{\varphi}_{n} M^{p}\right)=\operatorname{tr}\left(\hat{\varphi}_{n} M^{2 p}\right)=\sum_{j \geqq n+1}{ }^{n} t_{j}\right\| M^{p} e_{j} \|^{2}
$$

and so the assertion is true

Hereafter we shall write $\hat{\phi}=-\sum_{j} t_{j} P_{i}$, and the convergence is to be understood
ther in trace or $\beta^{*}$.

In summary, the pure states of a quantum mechanical system with $d$ degrees of freedom constitute a maximal nuclear space $\mathscr{W}[\mathrm{t}]$ of type $\mathscr{P} d \mathscr{W}$ decomposes into a countable sum of such spaces each of which is tvs isomorphic to $\mathscr{S}\left(\mathbf{R}^{d}\right)$. Such ari irreducible wave function space carries, a cyclic representation of the CCRs and the Fock-Cook condition is satisfied.

The algebra of observables is taken to be the complex unital *algebra $\mathcal{A}=\mathbf{L}^{+}(\mathscr{W})$, equipped with one of a number of topologies: $u, \sigma, \tau$, or $\beta$. It possesses a positive cone $\mathcal{A}_{+}$which is normal and generating for $u$, and $u$ is the order topology. With respect to $u, \mathcal{A}$ is a topological *-algebra.

The states are the normalized positive functionals on $\mathcal{A}$. All states are tracial (normal) and in the $\beta^{*}$-topology, $\mathcal{A}^{\prime} \cong \mathscr{W} \otimes \mathfrak{W}$ for irreducible $W$. 


\section{Expectations and instruments}

We assume that the reader is familiar with von Neumann's scheme for quantum measurements [22]. The following extract will suffice for our purposes. Let $\mathfrak{S}$ be the systemic Hilbert space and $a=\sum \alpha_{n} P_{n}$ a bounded self-adjoint operator on $\mathfrak{Q}$ with eigenvalues $\left(\alpha_{n}: n \geqq 1\right)$ and orthogonal one-dimensional projections $\left(P_{n}: n \geqq 1\right)$ onto the corresponding eigenvectors. A measurement of the observable $A$ in the state $\varphi$ will result in the occurrence of an eigenvalue of $A$. These eigenvalues are the only allowed values that can occur. If the eigenvalue $\alpha_{n}$ is observed, the measurement causes the collapse of the wave packet into the pure state represented by the "density matrix" $P_{n}$. This occurs with a probability $\varphi\left(\dot{P}_{n}\right)$. The Davies-Lew is theory [23-25] generalizes this scheme to symmetric operators with continuous spectra. In turn, our definitions $3.2,3.4$ below generalize their theory so as to be compatible with the algebraic formulation we have adopted. In what follows we shall be using Naimark's generalization of spectral theory which we quote in a form useful to us. As always, $\mathfrak{W}$ is the systemic separable Hilbert space.

Proposition 3.1: A generalized spectral family on $\mathfrak{S}$ is a one-parameier family $\left\{\mathscr{B}_{i}: t \in \mathbf{R}\right\}$ of operators $\left(0 \leqq \mathscr{B}_{t} \leqq 1\right)$ satisfying

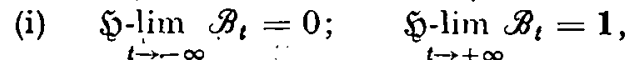

(ii) for all $t .<s, \quad \mathscr{B}_{t} \leqq \mathscr{B}_{s}$,

(iii) $\mathfrak{\mathfrak { Q }}-\lim _{\boldsymbol{t} \rightarrow+0} \mathscr{B}_{t+\varepsilon}=\mathscr{B}_{t}$.

$A$ positive operator-valued measure on $\mathfrak{Q}$ is a family $\mathscr{B}$ : $\operatorname{Bor}(\mathbf{R}) \rightarrow \mathbf{B}(\tilde{\mathfrak{\Phi}})$, where Bor (R) is the set of all Borel subsets of $\mathbf{R}$, and satisfying

(iv) $\mathscr{B}(\varnothing)=0 ; \quad \mathscr{B}(\mathbf{R})=\mathbf{1}$,

(v) for all $\Delta_{1} \subset \Delta_{2}, \quad \cdot \mathscr{B}\left(\Delta_{1}\right) \leqq \mathscr{B}\left(\Delta_{2}\right)$,

(vi) for every countable family of mutually disjoint Borel sets, $\left\{\Delta_{j}: j \geqq 1\right\}$,

$$
\mathscr{B}\left[\bigcup_{j \geqq 1}^{\cup} \Delta_{j}\right] \psi=\mathfrak{\mathfrak { Q } - \operatorname { l i m } _ { n \rightarrow \infty }} \sum_{1 \leqq j \leqq n} \mathscr{B}\left(\Delta_{j}\right) \psi,
$$

every $\psi \in \mathfrak{W}$.

Then just as for projection-valued spectral families, every generalized spectral family determines a positive operator-valued measure and conversely. The connexion is $\mathscr{B}(t)$ $=\mathscr{B}((-\infty, t])$.

If $b$ is a closed symmetric operator on $\mathfrak{W}$, there exists at least one generalized spectral family $\{\mathscr{B}(t): t \in \mathbf{R}\}$ such that'

(vii) $\operatorname{Dom}(b) \subset\left\{\psi: \int_{\mathbf{R}} t^{2}\langle\mathscr{B}(d t) \psi, \psi\rangle<\infty\right\}$,

(viii) for all $\psi \in \operatorname{Dom}(b)$ and all $\varphi \in \mathfrak{W}$,

$$
\begin{aligned}
& \langle b \psi, \varphi\rangle=\int_{\mathbf{R}} t\langle\mathscr{B}(d t) \dot{\psi}, \varphi\rangle, \\
& \|b \psi\|^{2}=\int_{\mathbf{R}} t^{2}\langle\mathscr{B}(d t) \psi, \psi\rangle .
\end{aligned}
$$

If $b$ is self-adjoint, then the family is projection-valued and unique. Moreover; equality then holds in (3.3. a). See [33, 34]. 
For a family of symmetric operators, defined on a common dense domain, we evidently require further conditions. Recall that every $b \in \mathcal{A}$ can be written as a linear combination of two symmetric operators: $\mathcal{A}=\mathscr{A}_{\mathrm{h}}+\dot{i} \mathcal{A}_{\mathrm{h}}$.

Definition 3.2 ; (a) An $\mathcal{A}$-measure is a generalized spectral family $\{\mathscr{B}(t): t \in \mathbf{R}\}$ on $\mathfrak{B}$ such that for all $\psi \in \mathscr{W}$,

$$
\int_{\mathbf{R}} i \mathscr{R}_{1}(d t) \psi=b \psi
$$

defines a symmetric operator $b \in \mathcal{A}_{\mathrm{h}}$. The integral is meant in the Riemann-Stieltjes sense, and converges in the $\mathfrak{Q}$-topology.

(b) An $(\mathcal{A}, \mathscr{W})$-measure is an $\mathcal{A}$-measure such that for all $\Delta \in \operatorname{Bor}(\mathbf{R})$,

$$
\mathscr{B}(\Delta),[\mathscr{W}] \subset \mathscr{W}
$$

We write $\mathscr{M}_{+}(\mathcal{A})$, resp. $\mathscr{M}_{+}(\mathcal{A}, \mathscr{W})$, for the set of all $\mathcal{A}$-measures, resp. $(\mathscr{A}, \mathscr{W})-$ measures.

Remark 3.3: (a) The inclusion $\mathscr{M}_{+}(A, \mathscr{Q}) \subset \mathscr{M}_{+}(\mathcal{A})$ is proper as can be seen from the coordinate multiplication operator $Q$ on $\mathscr{S}(\mathbf{R})$.

(b) DAVIES and LewIS use the term observable as synonymous with membership of $\mathscr{M}_{+}[\mathbf{B}(\mathfrak{Q})]=\mathscr{M}_{+}[\mathbf{B}(\mathfrak{Q}), \mathfrak{Q}]$. In contrast we reserve the term for membership of $\mathcal{A}$, c.f. $[23: 3.1 .1]$.

Aside from the continuous spectrum, two problems copfront us. The first is that $\mathscr{M}_{+}(\mathscr{A}, \mathscr{W}) \neq \mathscr{M}_{+}(\mathscr{A})$, and the second is that $\mathcal{A}[\mathfrak{u}]$ is not complete and not reflexive. This latter difficulty requires us to start our constructions by considering those linear maps on $\mathcal{A}$ which Davies calls expectations. [24]; by transposition we will get our notion of an instrument.

Definition 3.4: (a) An expectation is a map $Z$ : $\operatorname{Bor}(\mathbf{R}) \rightarrow \mathbf{L}_{+}\left(\mathcal{A}_{\mathrm{h}}\right)$ satisfying

(i) $Z(\emptyset)=0, Z(\dot{\Delta}) \geqq 0$ for all $\Delta \in \operatorname{Bor}$. $(\mathbf{R})$.

(ii) On $\mathscr{W}, Z(\mathbf{R})[1]=1$.

iii) For every countable family $\left\{\Delta_{j}: j \geqq 1\right\}$ of mutually disjoint Borel subsets, $Z$ is $\sigma$-additive in the sense

$$
\varphi\left\{Z\left(\bigcup_{j} \Delta_{j}\right)[b]\right\}=\sum_{j} \cdot \varphi\left\{Z\left(\Delta_{j}\right)[b]\right\},
$$

for all $\varphi \in \mathcal{A}_{\mathrm{h}}{ }^{\prime}$, all $b \in \mathcal{A}$.

$$
\int_{\mathbf{R}} t Z(d t)[1] \in \mathcal{A}
$$

where the Riemann-Stieltjes integral converges in the $\mathfrak{\$}$-topology.

(b) An instrument is a map $Q:$ Bor $(\mathbf{R}) \rightarrow \mathscr{L}\left(\mathscr{A}_{\mathrm{h}}^{\prime}\left[\sigma^{*}\right]\right)$ for which there is an expectation $Z$ whose transpose satisfies

$$
Z^{\imath}=Q
$$

We now consider some consequences of these definitions. The following characterization of an instrument is more or less immediate.

Lemma 3.5: Let $Q$ be an instrument. Then

(i) For every $\Delta \in$ Bor $(\mathbf{R}), Q(\Delta) \in \mathbf{L}_{+}\left(\mathcal{A}_{\mathbf{h}}{ }^{\prime}\right)$. 
(ii) $Q: \operatorname{Kor}(\mathbf{R}) \rightarrow \mathscr{L}_{+}\left(\mathcal{A}_{\mathrm{h}}{ }^{\prime}\left[\beta^{*}\right]\right)$.

(iii) For every countable family $\left\{\Delta_{j}: j \geqq 1\right\}$ of mutually disjoint Borel sets, $Q$ is $\sigma$-additive in the following sense: for every $\varphi \in \mathcal{A}_{\mathrm{h}}{ }^{\prime}$ and cvery $b \in \mathcal{A}$,

$$
Q\left(\cup_{j} \Delta_{j}\right)[\varphi](b)=\sum_{j}^{\Gamma} Q\left(\Delta_{j}\right)[\varphi](b) .
$$

(iv) $Q$ preserves normalization in the sense that for every $\varphi \in \mathcal{A}_{\mathrm{h}}{ }^{\prime}$,

$$
Q(\mathbf{R})[\varphi](\mathbf{1})=\varphi(\mathbf{1}) .
$$

Proof: Properties (iii), (iv) follow fromi (iii), (ii) respectively of Def. 3.4. Properties (i), (ii) follow from Prop. 2.14.(d)

We now embark on our analysis of the topological properties of instruments. In particular, we shall show that an instrument $Q$ is a map $\operatorname{Bor}(\mathbf{R}) \rightarrow \mathscr{L}_{+}\left(\mathcal{A}_{\mathrm{h}}{ }^{\prime}\left[\beta^{*}\right]\right)_{s}$ which is a bounded Radon measure. The subscript $s$ indicates that $\mathscr{L}_{+}$is equipped with the topology of simple convergence. We start by showing that the $\sigma$-additivity of (3.8) iniplies that $Q(\cdot)[\varphi]$ is $\beta^{*} \sigma$-additive.

Lem ma 3.6: An instrument is $\sigma$-additive as a map

$$
Q: \operatorname{Bor}(\mathbf{R}) \rightarrow \mathscr{L}_{+}\left(\mathcal{A}_{\mathrm{h}}{ }^{\prime}\left[\beta^{*}\right]\right)_{\mathrm{s}} \text {. }
$$

Proof: From (i), (iii) of Lemma 3.5 follows

$$
0 \leqq Q\left(\cup_{j \leqq n} \Delta_{j}\right)^{\prime}[\varphi] \leqq Q\left(\cup_{j \geqq n+1}^{\cup} \Delta_{j}\right)[\varphi] \leqq \cdots \leqq Q\left(\bigcup_{j} \Delta_{i}\right)[\varphi],
$$

for any positive functional $\varphi \in \mathcal{A}_{+}{ }^{\prime}$. By Cor. 2.16 it follows that

$$
\beta_{n}^{*}-\lim _{n} Q\left(\bigcup_{j \leqq n} \Delta_{j}\right)[\varphi]=\psi
$$

defines a functional $\psi \in \mathcal{A}_{\mathrm{h}}{ }^{\prime}$. We know by, (3.8) that

$$
\sigma_{n}^{*}-\lim _{n} Q\left(\bigcup_{j \leq n} \Lambda_{j}\right)[\varphi]=Q\left(\bigcup_{j} \Lambda_{j}\right)[\varphi]
$$

for all $\varphi \in \mathcal{A}_{h}{ }^{\prime}$. As $\sigma^{*} \leqq \beta^{*}$ we see that for all $\varphi \in \mathcal{A}_{+}{ }^{\prime}$

$$
\beta_{-n}^{*} \lim _{, n} Q\left(\bigcup_{j \leqq n} \Delta_{j}\right)[\varphi]=Q\left(\bigcup_{j} \Delta_{j}\right)[\varphi] \text {. }
$$

By linearity we may extend this to all $\varphi \in \mathcal{A}_{\mathrm{h}}{ }^{\prime}$, proving the assertion

The next step is to prove that $Q$ is inner regular.

Lemina 3.7: An instrument $Q$ is inner regular: for all $\Delta \in \operatorname{Bor}(\mathbf{R})$,

$$
Q(\Delta)=\underset{K \uparrow \Delta}{s-\operatorname{limi}} Q(K)
$$

where $s$ is the topology of simple convergence on $\mathscr{L}\left(\mathcal{A}_{\mathrm{h}}{ }^{\prime}\left[\beta^{*}\right]\right)$ and the limit is with respect to the filtering increasing compact subsets of $A$.

Proof: Recall that any positive Borel measure which is finite on compact subsets of a locally compact Hausdorff space, in which every open set is $\sigma$-compact. is regular [35: 2.18].

For any $p \in \mathscr{A}_{+}^{\prime}$ and $b \in \mathcal{A}_{+}$, the set map $m:$ Bor $(\mathbf{R}) \rightarrow \mathbf{R}, m(\Delta) \doteq Q(\Delta)[\varphi](b)$ is clearly a positive Borel measure on $\mathbf{R}$; and $\mathbf{R}$ is space of the aforementioned sort. Since $m$ is bounded: $m(\Delta) \leqq m(\mathbf{R})<\infty$, it follows that $m$ is regular, hence inner regular. In virtue of the $\sigma$-compactness of $\mathbf{R}$, inner regularity can be written as [36] 
$m(\Delta)=\lim m\left(K_{n}\right)$, where $\left(K_{n}: n \geqq 1\right)$ is an increasing sequence of compact subsets

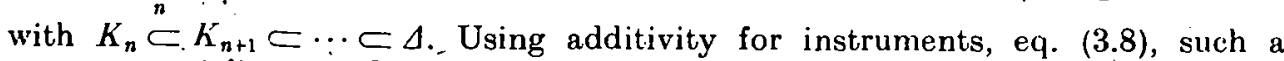
sequence satisfies $0 \leqq Q\left(K_{n}\right)[\varphi] \leqq Q\left(K_{n+1}\right)[\varphi] \leqq Q(\Delta)[\dot{\varphi}]$ for any $\varphi \in \dot{A}_{+}^{\prime}$. We can now apply Cor. 2.16 to get $Q(\Delta)[\varphi]=\beta^{*}$-lim $Q\left(K_{n}\right)^{\prime}[\varphi]$, and this extends linearly to all $\varphi \in \mathcal{A}_{\mathrm{h}}$ '. But this is what was to be shown, and we are done

In order that $Q$ be a bounded Radon measure it must certainly be bounded.

Lemma 3.8: Any instrument $Q$ is bounded for the tópology of simple convergence on $\mathscr{L}\left(\mathcal{U}_{\mathrm{h}}{ }^{\prime}\left[\beta^{*}\right]\right)$.

Proof: Now $\mathcal{A}$ is not reflexive but is identifiable with a total subset of $\mathscr{C}^{\prime \prime}\left[\mathrm{t}^{\prime}\right]$ $\otimes \mathscr{W}^{\prime}\left[\mathrm{t}^{\prime}\right]$, c.f. Props. 2.5, 2.14(b).

Let us show that for all $\varphi \in \mathcal{A}_{\mathrm{h}}^{\prime}$ the family $\{Q(\Delta)[\varphi]: \Delta \in \operatorname{Bor}(\mathbf{R})\}^{\prime}$ is $\sigma^{*}$-bounded. Write $\varphi=\varphi_{1}-\varphi_{2}$ with $\varphi_{1}, \varphi_{2} \in \mathcal{A}_{+}^{\prime}$ and let $b \doteq b_{1}-b_{2}$ with $b_{1}, b_{2} \in \mathcal{A}_{\mathrm{n}}$. Then, for all $\Delta$,

$$
|Q(\Delta)[\varphi](b)| \leqq \sum_{i, j} Q(\Delta)^{\prime}\left[\varphi_{i}\right]\left(b_{j}\right) \leqq \sum_{i, j} Q(\mathbf{R})\left[\varphi_{i}\right]\left(b_{j}\right)
$$

which is finite. Hence so is $\sup \{|Q(\Delta)[\varphi](b)|: \Delta \mid\}$, showing $\sigma^{*}$-boundedness. Tномаs has shown that if $E$ is an $F$-space and $m: X \rightarrow E$ an additive set function on a $\sigma$-algebra $X$, then a sufficient condition for $m$ to be bounded is that there exists a total subset $H \subset E^{\prime}$ such that $h \circ m$ is bounded for all $h \in H$ [37]. But this is precisely what we have shown; with $X=\operatorname{Bor}(\mathbf{R}), H=\mathcal{A}, E=\mathcal{A}_{\mathrm{h}}{ }^{\prime}\left[\beta^{*}\right]$ and $m=Z(\cdot)[\varphi]$, proving the lemma

Proposition 3.9: (a) Any instrument $Q$ is a continuous mapping

$$
Q: \mathbf{B}(\mathbf{R}) \rightarrow \mathscr{L}\left(\mathcal{A}_{\mathrm{h}}{ }^{\prime}\left[\beta^{*}\right]\right)_{\mathbf{s}},
$$

where $\mathbf{B}(\mathbf{R})$ is the normed space of bounded Borel functions equipped with the supremum norm: Thus $Q$ is a bounded Radon mapping in the sense of THOMAs [39].

(b) For every $\varphi \in \mathcal{A}_{\mathrm{h}}{ }^{\prime}, Q(\cdot)[\varphi]$ is a bounded Radon measiure.

Proof:, The first part is a consequence. of Iemmas 3.6-3.8 and [39]. For the second part we note that $\mathcal{A}_{\mathrm{h}}{ }^{\prime}\left[\beta^{*}\right]$ is reflexive, and then apply [38: Th. 5.3/p. 136 and Rem. 5.8/p. 139]

We sharpen this result, obtaining the desired property of instruments.

Theorem 3.10: Any instrument is a bounded Radon measure

$$
Q: \operatorname{Bor}(\mathbf{R}) \rightarrow \mathscr{L}_{+}\left(\mathcal{A}_{\mathrm{h}}{ }^{\prime}\left[\beta^{*}\right]\right)_{\mathrm{s}} \text {. }
$$

Proof: As $\mathcal{A}_{\mathrm{h}}{ }^{\prime}\left[\beta^{*}\right]$ is complete, the completion of $\mathscr{L}\left(\mathcal{A}_{\mathrm{h}}{ }^{\prime}\left[\beta^{*}\right]_{\mathrm{s}}\right.$ is $\mathbf{L}\left(\mathcal{A}_{\mathrm{h}}{ }^{\prime}\left[\beta^{*}\right]\right)_{\mathrm{s}}$ [40: p. 144]. Now $\mathscr{W}[\mathrm{t}]$ has a countable basis, say $\left\{e_{n}: n \in \mathbf{N}\right\}$. Therefore $\mathcal{A}_{\mathrm{h}}\left[\beta^{*}\right]$ has the countable basis $\left\{c_{n} \otimes c_{m}: n, m \in N\right\}$, c.f. [42: j. 23]. It follows that

$$
\mathbf{L}\left(\mathcal{A}_{\mathrm{h}}{ }^{\prime}\left[\beta^{*}\right]\right)_{\mathrm{s}}=\operatorname{Fl}_{\mathbf{N}} \mathcal{A}_{\mathrm{h}}{ }^{\prime}\left[\beta^{*}\right]
$$

with the product topology. Thus $\mathrm{L}\left(\mathcal{A}_{\mathrm{h}}{ }^{\prime}\left[\beta^{*}\right]\right)_{\mathrm{s}}$ is reflexive and Frechet $[40:$ p. 134; 6 : I.6.2., IV.5.8]. By [6: V.5.2], $\mathscr{L}_{+}\left(\mathcal{A}_{\mathrm{h}}{ }^{\prime}\left[\beta^{*}\right]\right)$ is a closed subspace of $\mathcal{L} \mathscr{L}\left(\mathcal{A}_{\mathrm{h}}{ }^{\prime}\left[\beta^{*}\right]\right)_{\mathrm{s}}$ and thus of $\mathbf{L}\left(\mathcal{A}_{\mathrm{h}}\left[\beta^{*}\right]\right)_{\mathrm{s}}$. Consequently $\mathscr{L}_{+}\left(\mathcal{A}_{\mathrm{h}}{ }^{\prime}\left[\beta^{*}\right]\right)$ is reflexive and Frechet. We then proceed exactly as in the proof of Prop. 3.9 above 
Corollary 3.11: (a) $\mathbf{L}_{+}\left(\mathcal{A}_{\mathrm{h}}{ }^{\prime}\left[\beta^{*}\right]\right)=\mathscr{L}_{+}\left(\mathcal{A}_{\mathrm{h}}{ }^{\prime}\left[\beta^{*}\right]\right)$.

(b) $\mathscr{L}_{+}\left(\mathcal{A}_{\mathrm{h}}{ }^{\prime}\left[\beta^{*}\right]\right)$ is a proper cone in $\mathscr{L}\left(\mathcal{A}_{\mathrm{h}}{ }^{\prime}\left[\beta^{*}\right]\right)_{s}$.

(c) $\mathscr{L}\left(\mathcal{A}_{\mathrm{h}}{ }^{\prime}\left[\beta^{*}\right]\right)_{\mathrm{s}}$ and $\mathscr{L}_{+}\left(\mathcal{A}_{\mathrm{h}}{ }^{\prime}\left[\beta^{*}\right]\right)_{\mathrm{s}}$ are nuclear.

Proof: (a): now follows from Prop. 2.14. (b) is true because $\mathcal{A}_{+}^{\prime}$ is total in $\mathcal{A}_{h}{ }^{\prime}\left[\beta^{*}\right]$, c.f. [ 6 , ibid]. (c) follows from the product representation eq. (3.14) above for $\mathbf{L}\left(\mathcal{A}_{\mathrm{h}}{ }^{\prime}\left[\beta^{*}\right]\right)_{\mathrm{s}}$ and the fact that $\mathcal{A}_{\mathrm{h}}{ }^{\prime}\left[\beta^{*}\right]$ is nuclear

Our next proposition brings a degree of physical interpretation by proving that an instrument uniquely determines an $(\mathcal{A}, \mathscr{W})$-measure. 'The reverse implication is one of non-uniqueness: a given $(\mathcal{A}, \mathscr{W})$-measure is determined by many instruments. This is consonant, with experience; there are many ways to measure the position of a particle.

Proposition 3.12: Given an instrument $Q$ there is a unique $(\mathcal{A}, \mathscr{W})$-measure $\Delta \rightarrow M(Q ; \Delta)$ determined by it. $M\left(Q_{:} \cdot\right)$ is given by extension from $\mathscr{W}$ to $\mathfrak{\mathcal { B }}$ of

$$
M(Q ; \Delta) f=Z(\Delta)[1] f, \quad(f \in \mathscr{W})
$$

where $Z$ is the expectation, unique, for which $Z^{t}=\dot{Q}$.

Given any $(\mathcal{A}, \mathscr{W})$-measire $\mathscr{B}$, there exist many instruments $Q$ such that $M(Q ; A)$ $=\mathscr{B}(\Delta)$. For example, for each state $\varphi$, the instrument

$$
Q(\varphi ; \Delta)=Z(\varphi ; \Delta)^{t} ; \quad Z(\varphi ; \Delta)[a]=\varphi(a) \mathscr{B}(\Delta) \cdot(a \in \mathcal{A}, \Delta \in \operatorname{Bor}(\mathbf{R}))
$$

is of this sort:

$$
1 .
$$

Proof: From the definition of an expectation it follows from (3.15) that $0 \leqq M(Q ; A) \leqq 1$ and so an extension of domain to- $\mathfrak{\mathfrak { Q }}$ is possible. For brevity we drop the $Q$ temporarily:

In the first plane $M$ must be shown to be a generalized spectral family. Only the $\sigma$-additivity is not obvious. To show this, let $f, g \in \mathscr{W}$ be arbitrary and let $P$ be the bounded $\mathfrak{G}$ operator $P(h)=\langle h, f\rangle g$. Taking $\varphi$ to be the functional determined by $P$ and with $b=1$, the additivity (3.6.c) of $Z$ yields

$$
\left\langle M\left(\cup_{j}, \Delta_{j}\right) f, g\right\rangle=\sum_{j}\left\langle M\left(\Delta_{j}\right) f, g\right\rangle \text {. }
$$

We now apply the following theorem of Thomas [38]: if $m:$ Bor $(\mathbf{R}) \rightarrow \mathfrak{G}$ is a set function such that $g \circ m$ is $\sigma$-additive for all $g \in \mathscr{W}$, then $m$ is $\sigma$-additive. Here we view $\mathscr{W}$ as a subset of the dual of $\mathfrak{W}$. With $m(\Delta)=M(\Lambda) f$, eq. (3.17) implies that $m$ is $\sigma$-additive. As $M(\Delta)$ is bounded, we can extend (3.17) to $g \in \mathfrak{g}$ and transpose $M(\Delta)$ to act on $g$. Repeating the above argument then implies that $M(\Delta) f$ is $\sigma$-additive for all $f \in \mathfrak{D}$. Hence $M$ is a generalized spectral measure.

Eq. (3.6.d) now implies that $M$ is an $\mathcal{A}$-neasure. To show that $M$ is an $(\mathcal{A}, \mathscr{W})$ : measure it suffices to show that $\varphi[M(\Delta)]$ is finite for all $\Delta$ and all $\varphi \in \mathcal{A}_{\mathrm{h}}{ }^{\prime}$. Using the spectral decomposition of nuclear operators, the cyclicity of the trace implies that

$$
\varphi[M(\Delta)]=\varphi(Z(\Delta)[\mathbf{1}])
$$

and as $Z$ is an expectation, $M(Q ; \cdot)$ is an $(\mathcal{A}, \mathscr{W})$-measure. Finally, the assertion that $Q(\varphi ; \cdot)$ is an instrument which determines $\mathscr{B}$ is obvious

Following Davies [24] we introduce the class of observables which can be measured. By this we mean that there exists at least one instrument which will give some information about the observable in question. 
Définition 3.12: (a) Given an $\mathscr{A}$-measure $\mathscr{B}$, let $\mathfrak{F}(\mathscr{B})$ be-the following set of functions of $b$ : .

$$
\left.\mathfrak{F}(\mathscr{B})=\sigma\left[\mathbf{B}(\mathfrak{\dagger})_{\mathrm{h}}, \mathscr{A}_{\mathrm{h}}{ }^{\prime}\right]-\nabla \dot{B}(\Delta): \Delta \in \operatorname{Bor}(\mathbf{R})\right\},
$$

where $v$ indicates linear span, and the closure of the span is in the indicated topology. Note that $B(\mathfrak{S})_{\mathrm{h}}$ and $\mathcal{A}_{\mathrm{h}}{ }^{\prime}$ constitute a dual pair.

We introduce a partial order on $\mathcal{A}_{+}(\mathcal{A})$ by setting

$$
\mathscr{B}<\mathscr{C} \text { if } \mathfrak{F}(\mathscr{B}) \subset-\mathscr{F}(\mathscr{C}) .
$$

Following [24]; we say that $\mathscr{B}$ gives less information than $\mathscr{C}$.

(b) An $\mathscr{A}$-measure $\mathscr{B} \in \mathscr{M}_{+}(\mathscr{A})$ is said to be physical if there exists an $(\mathscr{A}, \mathscr{W})$ measure $\mathscr{C} \in \mathscr{M}_{+}(\mathscr{L}, \mathscr{W})$ giving less information than $\mathscr{B}$, i.e.; $\mathscr{C}<\mathscr{B}$.

(c) An observable $b \in \mathcal{A}$ is said to be physical iff. it has a spectral representation by a physical $\mathcal{A}$-measure. If $\mathscr{C}<\mathscr{B}$ and $\mathscr{C}$ is a spectral representation of the observable $c \in \mathcal{A}$, we say that $c$ is a regularization of $b$.

(d). If $b \in \mathscr{A}$ is physical, and $\mathscr{B} \in \mathscr{M}_{+}(\mathscr{A})$ is any physical $\mathcal{A}$-measure spectrally representing $b$, then any instrument $Q$ such that $M(Q ; \cdot)<\mathscr{B}$ is said to be an instrument for measuring $b$.

Interpretation 3.13: Let $b \dot{\epsilon}$ A be a physical observable, and $Q$ an instrument for measuring it. For any state $\varphi$, the probability of obtaining an observation in $\Delta \in \operatorname{Bor}^{\prime}(\mathbf{R})$ on $Q$ is

$$
\varphi[M(Q ; \Delta)]=\operatorname{pr}(Q ; \varphi ; \Delta) .
$$

If such' an observation occurs, the state $\varphi$ collapses to the state

$$
\varphi \rightarrow \dot{Q}(\Delta)[\varphi] / Q(\Delta)[\varphi](\mathbf{1})
$$

the denominator providing normalization.

Remark 3.14: (a) An open question which would be of some interest to answer is the relation between physicality and the structure of $\mathscr{M}_{+}(\mathcal{A})$. In particular, do non-physical observables exist; when, if ever, does a regularization of an observable exist which is maximal with respect to the information partial order?

(b) Let $b=\sum \cdot \lambda_{n} P_{n} \in \mathcal{A}$ be self-adjoint such that $P_{n}[\mathscr{W}] \subset \mathscr{W}$ for all $n \geqq 1$. The associated spectral measure $\mathcal{P}$ is an $(\mathcal{A}, \mathscr{W})$-measure such that $\mathscr{P}(\bar{\Delta})=\sum P_{n}\left(\lambda_{n} \in \Delta\right)$. A "best" instrument for measuring $b$ is given by

$$
Q(\Delta)[\varphi]=\sum \varphi\left(P_{n} \cdot P_{n}\right)\left(\lambda_{n} \in \Delta\right) \text {. }
$$

This is the familiar collapse formula for the discrete case.

Of course the instrument $Q$ is also an instrument for measuring other observables, those $a \in \mathcal{A}$ for which $b<a$.

(c) If' $\mathscr{B} \in \mathscr{M}_{+}(\mathcal{A})$ is physical and projection valued, then any regularization of $\mathscr{B}$ is an abelian family.

\section{Composition and conditioning}

Let $Q_{1}, Q_{2}$ be instruments. Suppose we measure with $Q_{i}$ on a state $\varphi$ and obtain a positive result in the Borel region. $\Delta_{1}$; then we immediately measure with $Q_{2}$ 'on the new state. If we get a positive result in the region $\Delta_{2}$ the final outcome state will be the normalized form of

$$
Q_{21}\left(\Delta_{2} \times \Delta_{1}\right)[\varphi]=Q_{2}\left(\Delta_{2}\right)\left\{Q_{1}\left(\Delta_{1}\right)[\varphi]\right\}
$$


By virtue of our constructions, $Q_{12}\left(\Delta_{1} \times \Delta_{2}\right)[\varphi]$ is a positive functional for all Borel rectangles $\Delta_{1} \times \Delta_{2}$ and all positive functionals $\varphi$. Moreover it is the transpose of an $A$-stable map. However, we shall now show that whilst there is a unique extension to all Borel sets in Bor $\left(\mathbf{R}^{2}\right)$, the extension is not generally an instrument. Rather. it is the transpose of a map with range in. $W^{\prime} \otimes W^{\prime}$, the completion of $\mathcal{A}$.

Proposition 4.1: Let $Q_{12}$ be defined as in eq. (4.1) above. There exists a unique inner regular Radon measure

$$
Q_{12}: \operatorname{Bor}\left(\mathbf{R}^{2}\right) \rightarrow \mathscr{L}_{+}\left(\mathscr{A}_{\mathrm{h}}{ }^{\prime}\left[\beta^{*}\right]\right)_{9}
$$

such that for all Borel rectangles expression (4.1) results. In general, $Q_{12}$ is the transpose of a map $Z_{12}$ : Bor $\left(\mathbf{R}^{2}\right) \rightarrow \mathbf{L}_{+}\left(\left[\mathscr{W}^{\prime}\left(\mathrm{t}^{\prime}\right)(\dot{\otimes}) \mathscr{W}^{\prime}\left(\mathrm{t}^{\prime}\right)\right]_{\mathrm{h}}\right)$ and is not, therefore, an instrument.

Before proving this proposition we present two preliminary lemmas. Note first that we have taken the liberty of implicitly extending all our previous definitions and construction of $\S 3$ from Bor $(\mathbf{R})$ to Bor $\left(\mathbf{R}^{2}\right)$. Obviously all the results remain true.

The measure $Q_{12}$ will be referred to as the compose of $Q_{1}$ and $Q_{2}$.

Lemma 4.2: Let $\left\{T_{j}: j \in J\right\}$ be an upper bounded and upward directed net in $\mathscr{L}\left(\mathcal{A}_{n}{ }^{\prime}\left[\beta^{*}\right]\right)_{s}$. Then the net converges in the simple topology to its supremum:

$$
T^{\prime}=\sup \left\{T_{j}: j \in J\right\}=\underset{J}{\operatorname{sim}} T_{i} \cdot
$$

Proof: By Prop. 2.17; $\left\{T_{j} \varphi: j \in J\right\}$ converges to its supremum for each $\varphi \in \mathcal{A}_{+}$'. As $\mathscr{L}\left(\mathcal{A}_{\mathrm{h}}{ }^{\prime}\left[\beta^{*}\right]\right)$ is s-complete, the result follows

Lemma 4.3: $\mathscr{L}_{+}\left(\mathcal{A}_{\mathrm{h}}{ }^{\prime}\left[\beta^{*}\right]\right)_{\mathrm{s}}$ is a topological algebra under the product $S, T \rightarrow S T . I f$ $\left\{T_{j}: j \in J\right\}$ is a net as in Lemma 4.2, $S T_{j} \rightarrow S^{\prime} T^{\prime}$ and $T_{j} S \rightarrow T S$ for all $S$.

\section{Proof: Obvious}

Proof of Proposition 4.1: The existence of a unique extension of $Q_{12}$ to Bor $\left(\mathbf{R}_{\mathrm{f}}^{2}\right)$, satisfying the stated conditions follows from applying Lemma (4.3) to Theorem (1.7) of [42]. That $\mathcal{Q}_{12}$ is not an instrument in general follows from the non reflexivity of $\mathcal{A}$

The fact that $Q_{12}$ is not an instrument is 'mildly disturbing. However, a strong case can be made, on operational grounds, for defining expectations and instruments. not on all Borel sets, but only on intervals, perhaps only on finite intervals. It is difficult to imagine, e.g., how one would measure the position of a particle within an extremely wild Borel set, nor even why one would wish to. Be that as it may, it seems useful to introduce the notions of preand post instruments and expectations.

Definition 4.4: (a) A pre-expectation is a map Z: $\mathbf{Z} d \boldsymbol{d} \rightarrow \mathbf{L}_{+}\left(\mathcal{A}_{\mathrm{h}}\right)$ satisfying the conditions $\mathrm{i}-\mathrm{iv}$ of an expectation (Definition 3.4 ) save that $\mathbf{P}^{d}$ is the ring generated by all polyintervals in $\mathbf{R}^{d}$, so the families of disjoint sets must satisfy $\Delta_{j} \in \mathbf{P}^{d}$ and $\cup A_{j} \in \mathbf{P}^{d}$. As well, the integral eq. (3.6.4.) is over. $\mathbf{R}^{d}$.

A post-expectation is a map $Z:$ Bor $\left(\mathbf{R}^{d}\right) \rightarrow \mathbf{L}_{+}\left(W^{\prime} \otimes W^{\prime}\right)$, where $W^{\prime}$ has its strong dual topology, and satisfying conditions $\mathrm{i}-\mathrm{iii}$, with iv replaced by

$$
\int_{\mathbf{R}^{c}} t_{1} t_{2} \ldots t_{d} Z\left(d t_{1} d t_{2} \ldots d t_{d}\right)[1] \in \mathscr{W}^{\prime} \otimes W^{\prime} .
$$

(b) A pre-instrument is a map $Q: \mathbf{P}^{d} \rightarrow \mathscr{L}\left(\mathcal{A}_{\mathrm{h}}{ }^{\prime}\left[\sigma^{*}\right]\right)$ which is the transpose of a preexpectation. Similarly for a post-instrument, with domain $\operatorname{Bor}\left(\mathbf{R}^{d}\right)$. 
We shall not examine the general consequences of these definitions in this paper save for three remarks:

The first is that we could have demanded only finite additivity for pre-expectations and pre-instruments. However, one could always extend uniquely to $\sigma$-additivity, cf. [46] and references therein.

The second remark is that every pre-instrument has a unique continuous extension to a post-instrument. The quality of being an instrument seems delicate.

Thirdly, every instrument appearing in this paper compose with every other such instrument to give an instrument. We take this to be a result of special circumstances. Nonetheless, we do not have an example of two instruments which compose to a post-instrument which is not an instrument.

Davies and Lewis also defined joint distributions and conditioned observablés.

Proposition 4.5: Let $Z_{1}, Z_{2}$ be expectations, $\mathscr{B}_{1}, \mathscr{B}_{2}$ their respective $(\mathcal{A}, \mathscr{W})$-measures, and $Q_{1}, Q_{2}$ the respective instruments.

The joint distribution of $Z_{2}$ following $Q_{1}$ is defined to be the map

$$
Z_{21}: \operatorname{Bor}\left(\mathbf{R}^{2}\right): \mathcal{L}\left(\mathscr{W}^{\prime} \otimes W^{\prime}\right) ; \quad Z_{21}(w)=Q_{2}(w)^{t} .
$$

Then $Z_{21}$ is a post-expectation whose marginal distributions satisfy

Hence

$$
Z_{21}(\mathbf{R} \times \Delta)=Z_{1}(\mathbf{R}) Z_{2}(\Delta) ; \quad Z_{21}(\Delta \times \mathbf{R})=Z_{1}(\Delta) Z_{2}(\mathbf{R}) .
$$

$$
Z_{21}(\mathbf{R} \times \dot{\Delta})[1]=Z_{1}(\mathbf{R})\left[\mathscr{B}_{2}(\Delta)\right] ; \quad Z_{21}(\Delta \times \mathbf{R})[1]=\mathscr{B}_{1}(\Delta)
$$

are $(\mathcal{A}, \mathscr{W})$-measures.

The map $\Delta \rightarrow Z_{1}(\mathbf{R})\left[\mathscr{B}_{2}(\Delta)\right]$ is the $(\mathcal{A}, \mathscr{W})$-meaisure $\mathscr{B}_{2}$ conditioned by the measurement of $\mathscr{B}_{1}$ with the instrument $Z_{1}$, cf. [25: Th. 3].

To end this section let us note that instruments generally have no repeatability properties. We have not examined the $\varepsilon$-repeatability properties of our instruments, cf. [25].

\section{A class of instruments on $\mathscr{F}(\mathbf{R})$}

In this section we consider the system $\mathscr{W}=\mathscr{F}(\mathbf{R})$, with one degree of freedom: Our principle result is the explicit formula for a family of instruments which will measure the basic quantum mechanical operators with some degree of accuracy. We note that the basic formula was proposed by DaviEs [23] as a covariant approximate position instrument. What is new here is that we consider the family as labelled by the normalized elements of $\mathscr{S}(\mathbf{R})$ and show that the result is an instrument in our sense, i.e.; with reference to the algebra $\mathcal{A}$. We also show that the formula is valid for more operators than the position and that the compose of any two instruments is an instrument. Our principal result is this.

Proposition 5.1: Let $a \in \mathcal{A}_{\mathrm{h}}$ stund for any of the essentially self-adjoint operators $Q ; P=-i D$, or $H=P^{2}+V(Q)$, where $x \rightarrow V(x)$ is $C^{\infty}$ and, together with all of its, derivatives, is bounded. .

To each $f \in \mathscr{S}(\mathbf{R})$ the associated map

$$
Z[a ; f ; \Delta](b)=\int_{\Delta} f_{s}(a)^{*}, b f_{s}(a) d s,
$$


where $f_{s}(a)$ is defined by the spectral calculus, with $f_{s}(x)=f(x-s)$ :

$$
\begin{aligned}
& f_{0}(a)=\int_{\mathbf{R}} f(x-s) E(d x), \\
& a=\int_{\mathbf{R}} x E(d x),
\end{aligned}
$$

is an expectation. By an abuse of notation we are not distinguishing a $\in \mathcal{A}_{\mathrm{h}}$ from its closure $a^{* *}$. The function $f$ is reguired to be normalized:

$$
\|f\|^{2}=1
$$

Before passing to the proof of this proposition let us make a few remarks about the physical interpretation of $Z[a ; f ; \cdot]$ or, equivalently, its corresponding instrument $Q[a ; f ; \cdot]=Z[a ; f ; \cdot]^{t}$.

Were it possible to build a perfect instrument, and it is not, it would be the simple generalization of the.discrete formula, viz,

$$
Z_{\infty}[a ; \Delta](b)=\int_{\Delta} E(s) b E(s) d s
$$

One reason why $Z_{\infty}$ is not an instrument is that it "chops off the incoming wave functions too sharply" at the boundaries of $\Delta$. As it is required that " $\mathscr{F}(\mathbf{R})$ in, $\mathscr{P}(\mathbf{R})$ out", we.must smooth $Z_{\infty}$ out. Hence regularizing $Q$ with $f$. More precisely:

Corollary 5.2: The $(\mathcal{A}, \mathscr{P})$-measure $M(Q ; \Delta)$ corresponding to the instrument $Q \equiv Q[a ; f ; \cdot]$ is

$$
M(Q ; \Delta)=\int F * \chi_{\Delta}(x) E(d x)=F * \chi_{\Delta}(a),
$$

with $F(x)=|f(x)|^{2}$, and convolution is meant.

This formula was given by Davies; that it defines an $(\mathcal{A}, \mathscr{W})$-measure is a consequence of our general theory, Prop. 3.12, once we show $Z$ to be an expectation.

One measure of the goodness of an instrument, probably not a useful measure, is the difference between the instrument $(\mathcal{A}, \mathscr{W})$-measure $M$ and the $\mathcal{A}$-measure in question, here $E$. If we consider, in the $\mathbf{B}(\mathfrak{Q})$ norm,

$$
\left\|F * \chi_{\Delta}(a)-E(\Delta)\right\|,
$$

we see that this vanishes for $F=\delta$. As $F \in \mathscr{H}$ and $\mathscr{S}$ is dense in $\mathscr{S}^{\prime}$, choosing $F$ close to. $\delta$ in some way, makes $Q$ a good instrument. Similarly, choosing $F$ close to the constant function makes $Q$ into a poor instrument.

Now to the proof of the proposition. The proof is rather long with the appearance of numerous inequalities involving the $\mathscr{F}(\mathbf{R})$ seminorms. These are relatively straightforward, so we have not given the full derivation.

In all that follows we shall be using four sets of seminorms, namely, in an obvious notation,

$$
\begin{aligned}
& \|f\|_{n, m ; \infty}=\left\|t^{n} D^{m} f\right\|_{\infty} ; \quad\|f\|_{k ; \infty}=\max \left\{\|f\|_{n ; m ; \infty}: 0 \leqq n, m \leqq k\right\}, \\
& \|f\|_{n, m ; 2}=\left\|\ell^{n} D^{m} f\right\|_{2} ; \quad\|f\|_{k ; 2}=\max \left\{\|f\|_{n, m ; 2}: 0 \leqq n, m \leqq k\right\}
\end{aligned}
$$

Each set defines the usual topology on $\mathscr{S}$. The index ranges are $n, m, k \in \mathbf{N}$.

We shall use the following estimates, which are too well known to merit proof for $Q, P ;$ for $H$ see [43]. 
Lemina 5. 3:

(i) $Q^{m} P^{n} \mathrm{e}^{i a Q}=\mathrm{e}^{i a Q} \sum_{k \leqq n}\left(\begin{array}{l}n \\ k\end{array}\right) a^{n-k} Q^{m} P^{k}$.

(ii) $\quad Q^{m} P^{n} \mathrm{e}^{i b P}=\mathrm{e}_{1}^{i b P} \sum_{k \leqq m}\left(\begin{array}{c}m \\ k\end{array}\right) b^{m-k} Q^{k} P^{n}$

(iii) $Q^{n} e^{-i t H}=\mathrm{e}^{-i t H} Q^{n}+i \int_{0} \mathrm{e}^{-i(t-s) H}\left[H, Q^{n}\right] \mathrm{e}^{-i s H} d s$,

with

$$
\left[H, Q^{n}\right]=2 i n P Q^{n-1}+n(n-1) Q^{n-2} .
$$

(iv). $\quad\left\|\mathrm{e}^{i a \theta} f\right\|_{m, n ; 2} \leqq \sum_{k \leqq n}\left(\begin{array}{l}n \\ k\end{array}\right)|a|^{n-k}\|f\|_{m, k ; 2}$

$$
\leqq(1+|a|)^{n}\|f\|_{m, 2} \text {. }
$$

(v) $\quad\left\|\mathrm{e}^{i b P} f\right\|_{m ; n ; 2} \leqq \sum_{k \leqq m}\left(\begin{array}{c}m \\ k\end{array}\right)|b|^{m-k}\|f\|_{k, n ; 2}$

$$
\leqq(1+|\grave{b}|)^{m}\|f\|_{n ; 2} \text {. }
$$

(vi) $\quad\left\|\mathrm{e}^{-i t /} f\right\|_{2 n ; 2} \leqq c_{n}(1+|t|)^{n}\|f\|_{2 n ; 2}$.

The Fourier transform of the characteristic function of a Borel set is a tempered distribution, as we now show. This will provide a useful estimate in what follows.

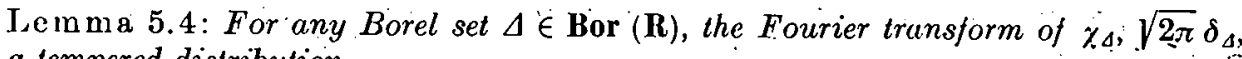
is a tempered distribution.

Proof: It is sufficient to show that $\chi_{\Delta} \in \mathscr{S}^{\prime}$. Simply, for all $f \in \mathscr{S}$, multiplying and dividing by $1+t^{2}$ gives $\left|\chi_{\Delta}(f)\right| \leqq \sup _{t}\left|\left(1+t^{2}\right) f(t)\right| \int_{\Delta}\left(1+t^{2}\right)^{-1} d t \leqq \pi\|f\|_{2 ; \infty}$

The immediate consequence is that for each Borel set $\Delta$ there exists an index $M$ and constant $c$, depending on $\Delta$, such that for every $f \in \mathscr{S}$,

$$
\left|\delta_{\Delta}(f)\right| \leqq c\|f\|_{m ; \infty} .
$$

For definiteness let us now specialize to $a=Q$. The calculations are quite similar for the other cases $a=P, H$.

Lemma 5.5: Let $f$ be the function labelling the instrument, and $g$ its Fourier transform; hence $g_{t}$ is the transform of $f_{t}$. Let $b \in \mathcal{A}$ and write

$$
b_{t}=\mathrm{e}^{i t Q} b \mathrm{e}^{-i t Q}, \quad(t \in \mathbf{R})
$$

so that $b_{t} \in \mathcal{A}$ for each $t \in \mathbf{R}$. Let $\varphi, \psi \in \mathscr{S}$ be arbitrary and consider the function.

$$
s \rightarrow h_{t}(s)=g_{t}(s)\left\langle\mathrm{e}^{i s 0} b_{t} \varphi, \psi\right\rangle_{N ; 2},
$$

where

$$
\langle\varphi, \psi\rangle_{N ; 2}=\max _{0 \leqq a . b \leqq N_{-}}\left\langle Q^{a} D^{b} \varphi, \psi\right\rangle,
$$

and so, e.g.

$$
\left|\langle\varphi, \psi\rangle_{N ; 2}\right| \leqq\|\psi\|\|\varphi\|_{N ; 2}
$$


Then $h_{t} \in \mathscr{S}(\mathbf{R})$, with

$$
\left\|\dot{h}_{t}\right\|_{M ; \infty} \leqq 2^{N+3 M}(1+|t|)^{N+2 M}\|\psi\|\left\|b_{t} \varphi\right\|_{N+M ; 2}\|g\|_{N+2 M ; \infty} .
$$

Proof: With eq. (5.7.e) and (5.11.b) we find that

Then

$$
\left|\left\langle\mathrm{e}^{i s Q} b_{\imath} \varphi, \psi\right\rangle_{N ; 2}\right| \leqq\|\psi\|(1+|s|)^{N}\left\|b_{l} \varphi\right\|_{N ; 2} .
$$

$$
\begin{aligned}
\left\|h_{t}\right\|_{M ; \infty} & \leqq \max _{u, v \leqq M} \sum_{v o=0}^{v}\left(\begin{array}{c}
v \\
w
\end{array}\right) \sup _{. s}\left|s^{u} D^{v-w} g_{t}(s)\left\langle\mathrm{e}^{i s Q} b_{t} \varphi, \psi\right\rangle_{N+M ; 2}\right| \\
& \leqq\|\psi\|\left\|b_{t} \varphi\right\|_{N+M ; 2} \max _{u, v \leqq M} \sum_{w=0}^{v} \sup _{s}\left|s^{u}(1+|s|)^{N+M} \dot{D}^{v-w} g_{t}(s)\right| .
\end{aligned}
$$

We now use

$$
|s|^{u}(1+|s|)^{N+M} \leqq 2^{N+M}|s|^{N+2 M} \text { and }:\left\|g_{t}\right\|_{P ; \infty} \leqq(1+|t|)^{P}\|g\|_{P ; \infty} ;
$$

the result follows

It will be necessary to find an estimate in $t$ for $\left\|b_{t}\right\|_{N+M ; 2}$.

Lem ma 5.6: For every $b$ and $N+M$ there, exists an index $P$ and a positive constant $c_{1}$ such thai for all $t \in \mathbf{R}$ and all $\varphi \in \mathscr{S}$

$$
\left\|b_{t} \varphi\right\|_{N+M ; 2} \leqq c_{1}(1+|t|)^{N+M+P}\|\varphi\|_{P ; 2} \cdot
$$

Proof: First of all

$$
\left\|b_{\imath} \varphi\right\|_{N+M ; 2} \leqq(1+|t|)^{N+M}\left\|\dot{b} \mathrm{e}^{-i t Q} \varphi\right\|_{N+M ; 2} .
$$

, The continuity of $b$ gives $\|b \psi\|_{N+M ; 2} \leqq c_{1}\|\psi\|_{P ; 2}$ and with $\psi=\mathrm{e}^{-i 10} \varphi$ the result is immediate

\section{Proof of Proposition 5.1: We must show that}

$$
\|Z(\Delta)[b] \varphi\|_{N ; 2}=\sup \left\{\left|\langle Z(\Delta)[b] \varphi, \psi\rangle_{N ; 2}\right|: \psi \in \dot{S}^{\prime} \mathscr{P},\|\psi\|_{,}=1\right\}
$$

is finite for all $\Delta, b, \varphi$. Using Fourier transforms,

where

$$
\left|\langle Z(\Delta)[b] \varphi, \psi\rangle_{N ; 2}\right| \leqq \int_{\mathbf{R}}|g(t)||J(t)| d t
$$

$$
J(t)=\int_{\mathbf{R}} g_{t}(s) \delta_{\Delta}(s)\left\langle\mathrm{e}^{i s} b_{l} \varphi, \psi\right\rangle_{N ; 2} d s .
$$

We have changed váriables $s-t \rightarrow \dot{s}$ in this expression. Now we observe that $J(t)=\delta_{\Delta}\left(h_{\imath}\right)$. Using eq. (5.8); (5.12), and (5.13) yields

$$
|J(t)| \leqq c_{2}\|\psi\|(1+|i|)^{2 N+3 M+P}\|\varphi\|_{P ; 2}\|g\|_{N+2 M ; \infty} .
$$

But then

$$
\|Z(\Delta)[b] \dot{\varphi}\|_{N ; 2} \leqq c_{3}\|\dot{\varphi}\|_{P ; 2}\left(\|g\|_{N+2 M+2 ; \infty}\right)^{2}
$$

This shows that for every Borel set $\Delta \in \operatorname{Bor}(\mathbf{R}), Z(\Delta)$ is a linear map from $\mathcal{A}_{\mathrm{h}}$ to itself. It is obvious that the positive cone is stable under $Z(\Delta)$. The normalization condition is

$$
Z(\mathbf{R})[\mathbf{1}]=\int_{\mathbf{R}} f_{s}(Q)^{*} f_{s}(Q) d s=\mathbf{1}
$$


As $f_{s} \in \mathscr{J}$ for each $s \in \mathbf{R}$ and $f_{s}(Q)$ is bounded, the spectral calculus for bounded operators applies, changes of order of integration are allowed, and as $\left\|f_{x}\right\|^{2}=1$, the condition is verified.

Now we shall prove the requisite $\sigma$-additivit $\nabla$, eq. (3.6.c). Let $\varphi \in \mathcal{A}_{+}^{\prime} ; b \in \mathcal{A}_{+}$be arbitrary and $\left\{\Delta_{j}: j \geqq 1\right\}$ be a family of mutually disjoint Borel sets with $\bigcup \Delta_{j}$ $\epsilon$ Bor (R). We use the spectral representation of $\hat{\phi}$ and Prop. (2.18) to get

$$
\varphi(Z(\Delta)[b])=\sum_{n \geqq 1} t_{n}\left\langle Z(\Delta)[b] e_{n}, e_{n}\right\rangle
$$

for all Borel sets $\triangle$. The specific form of $Z$ yields

$$
\left\langle Z(\Delta)[b] e_{n}, e_{n}\right\rangle=\int_{\Delta}\left\langle f_{w}(Q) * b f_{w}(\dot{Q}) e_{n}, e_{n}\right\rangle d w
$$

The integral is $\sigma$-additive, cf. [44: Exercise (29.6)], and so, changing back to the Z form,

$$
\left\langle Z\left(\bigcup_{j} \Delta_{j}\right)[b] e_{n}, e_{n}\right\rangle=\sum_{j}\left\langle Z\left(\Delta_{j}\right)[b] e_{n}, e_{n}\right\rangle
$$

for each $n$. By [45: Theorem (8.3)] we can interchange summation order, to get

$$
\varphi\left(Z\left(\bigcup_{j}^{\cup}, \Delta_{j}\right) \cdot[b]\right)=\sum_{j} \sum_{n} t_{n}\left\langle Z\left(\Delta_{j}\right)[b] e_{n}, e_{n}\right\rangle=\sum_{i j} \varphi\left(Z\left(\dot{\Delta_{j}}\right)[b]\right) .
$$

By linearity this extends to all $\varphi \in \mathcal{A}_{\mathrm{h}}{ }^{\prime}, b \in \mathcal{A}_{\mathrm{h}}$, and so $Z$ is $\sigma$-additive in the $\sigma$-topology.

Finally we shạll show that $\int_{\mathbf{R}} t(d t)[1]$ is an element of $\mathcal{A}$, whence $Z$ will have been proved to be an expectation. By the spectral calculus, as mentioned above, for all $\varphi, \psi \in \mathscr{S}$.

$$
\therefore\langle Z(\Delta)[1] \varphi, \psi\rangle=\left\langle F_{\Delta}(Q) \varphi, \psi\right\rangle, \quad \Delta \in \operatorname{Bor}(\mathbf{R})
$$

where $F_{\Delta}(t)=\int_{\Delta}|f(t-s)|^{2} d s$. As $0 \leqq F_{\Delta}(t) \leqq F_{\mathbf{R}}(t)$ and $F_{\mathbf{R}}(t)=\left\|f_{2}\right\|^{2}=1$; it follows that $0 \leqq F_{\Delta}(Q) \leqq 1$. We can continuously extend $F_{\Delta}(Q)$ from $\mathscr{S}$ to all of $L^{2}(\dot{\mathbf{R}})$, whereby it is easy to see that, with

$$
\left\langle F_{d}(Q) \varphi, \psi\right\rangle \fallingdotseq \int_{d}\left\langle f_{w}(Q)^{*} f_{w}(Q) \varphi, \psi\right\rangle d w, \quad\left(\varphi, \psi \in L^{2}\right)
$$

$\Delta \rightarrow F_{\Delta}(Q)$ is a generalized spectral family. This family defines a symmetric operator, call it

$$
\mathscr{X}=\int_{\mathbf{R}} t F_{d t}(Q) \supset \int_{\mathbf{R}} t Z(d t)[1]
$$

Now we must show that $\mathscr{X} \in \mathcal{A}$.

Firstly,

$$
\mathscr{H}(\mathbf{R}) \subset \operatorname{Dom}(\mathscr{X})^{\prime}=\left\{\varphi \cdot L^{2}: \int_{\mathbf{R}} t^{2}\left\langle F_{d t}(Q) \varphi, \varphi\right\rangle<\infty\right.
$$

But if $\varphi \in \mathscr{P}$, a simple estimate gives

$$
\left|\int_{\mathbf{R}} t^{2}\left\langle F_{d t}(Q) \dot{\varphi}, \varphi\right\rangle\right|=\mid \int_{\mathbf{R}}\left(\int_{\mathbf{R}} t^{2}(f(s-t))^{2} d s\langle E(d t) \varphi, \dot{\phi}\rangle \mid\right.
$$

which is finite.

$$
\leqq\left(\|f\|_{2 ; 2}\right)^{2}\left\langle\left(Q^{2}+4 Q+1\right) \varphi, \varphi\right\rangle
$$


Secondly we show that for all $\varphi \in \mathscr{S}(\mathbf{R}), \mathscr{X} \varphi \in \mathscr{S}(\mathbf{R})$. To see this, let $\varphi, \psi \in \mathscr{P}$ be arbitrary. Then it is easy to show that

$$
\langle\mathscr{X} \varphi, \psi\rangle=\langle\varphi ; \psi\rangle \int_{\mathbf{R}} t|f(t)|^{2} d t+\langle Q \varphi, \psi\rangle .
$$

But then by considering $\left\langle\mathscr{X} \varphi, Q^{m} D^{n} \psi\right\rangle$ and taking the supremum over $\psi,\|\psi\|=1$, we find that

$$
\|\mathscr{X} \varphi\|_{m, n ; 2} \leqq\|\varphi\|_{m, n ; 2} \int_{\mathbf{R}} t|f(t)|^{2} d t+\|Q \varphi\|_{m, n ; 2}
$$

and so $\mathscr{X} \in \mathcal{A}_{\mathrm{h}}$, proving the proposition

Corollary 5.7: The $(\mathcal{A}, \mathscr{J})$-measure determined by $Z[f ; Q ; \cdot]$ is

$$
\Delta \rightarrow\left[\chi_{\Delta} *|f|^{2}\right](Q) \text {. }
$$

As this is bounded for all $\Delta$, it is an element of $\mathfrak{F}(E)$, cf. eq. (3.18.a), and so $Q[f ; Q ; \cdot]$ is an instrument for measuring $Q$

The reader will note that we have not proved the proposition for $P$ and $H$. In view of Lemma 5.3, the reader will. see that these calculations are entirely similar. Details will be found in [47].

As we mentioned previously, because $\mathcal{A}[u]$ is incomplete, a pre-instrument may, in general, extend to a post-instrument. This applies in particular to the compose of two instruments. We now have a class of instruments $Q[f ; a ; \Delta]$ for $f \in \mathscr{f}(\mathbf{R})$, $a=Q, P, H$. By computing the compositions explicitly we shall show that the compose of any two, hence any finite number, of these instruments is an instrument. As regards the implications for the general case, we believe that this result is special, and depends on the translation covariance of these instruments and various special properties.

To write out the proof in any detail would be longer than the proof that $Q$ is an instrument. Moreover, it depends upon estimates obtained precisely as for Prop. 5.1, but "doubled up". For these reasons we choose simply to state the required estimates and refer to [48] for details. The form of the estimates will, we feel, be convincing.

Proposition 5.8: Let $Z_{j}\left[f_{j}, a_{j} ; \Delta\right](j=1,2)$ be expectations of the type described in Proposition 5.1. Then $Z_{1} \circ Z_{2}$ is an expectation.

Proof: We introduce the following notation. By $U_{j}$ 'we mean the one-paraneter unitary group on $L^{2}(\mathbf{R})$ generated by the observable $a_{j}$, and by $\alpha_{j}$ the corresponding automorphism group of $\mathcal{A}$. By $g_{12}$ we mean the Fourier transform of $f_{1} \otimes f_{2}$, and for any $\theta \in \operatorname{Bor}\left(\mathbf{R}^{2}\right), \delta_{\theta}$ is $(2 \pi)^{-1}$ times the Fourier transform of $\chi_{\theta}$.

$J$ ust as for $Z$ we can use Fourier transforms to show that for all Borel rectangles, all $\varphi, \psi \in \mathscr{F}(\mathbf{R}), b \in \mathcal{A}, N \geqq 0$

$$
\begin{aligned}
& \left\langle Z_{12}\left(\Delta_{1} \times \Delta_{2}\right)[\dot{b}] \varphi, \psi\right\rangle_{N ; 2} \\
& =\int_{\mathbf{R}^{2}} \dot{g}_{12}(\xi) \cdot\left(\int_{\mathbf{R}^{2}}\left[e^{\xi \cdot \mathbf{D}} g_{12}\right]^{*}(\eta) h(\xi ; \eta) \delta_{\Delta_{1} \times \Delta_{2}}(\eta) d \eta\right) d \xi .
\end{aligned}
$$

An obvious vector notation has been introduced, so that, e.g., $\mathbf{D}=\left(\partial / \partial \eta_{1}, \partial / \partial \eta_{2}\right)$. We have also introduced the function

$$
h(\xi ; \eta)=\left\langle\alpha_{2}\left(\dot{\xi}_{2}\right) U_{2}\left(\eta_{2}\right) U_{1}\left(\eta_{1}\right) \alpha_{1}\left(\xi_{1}\right)[b] \varphi, \psi\right\rangle_{N ; 2} .
$$


Let us specialize to $a_{1}=Q$ and $a_{2}=H$ for definiteness. Our first estimate is that for any $u \geqq 0$ there exist indices and a constant so that

$$
\left\|\left[\mathrm{e}^{\xi \cdot \mathbf{D}} g_{12}\right] * h(\xi ; \cdot)_{u ; \infty} \leqq C_{1}\left(1+\left|\xi_{1}\right|\right)^{n_{1}}\left(1+\left|\xi_{2}\right|\right)^{\dot{n}_{2}}\right\| \psi\|\| \varphi\left\|_{n_{3} ; 2}\right\| g_{12} \|_{n_{i} ; \infty}:
$$

For any set $\theta \in$ Bor $\left(\mathbf{R}^{2}\right)$ and any function $G$ such that its Fourier transform $\tilde{G} \in L^{1}\left(\mathbf{R}^{2}\right), \quad\left|\delta_{\theta}(G)\right| \leqq\|\widetilde{G}\|_{1}$. Note that the bound is independent of $\theta$. After some I manipulation we find that there exist indices and a constant so that

$$
\begin{aligned}
& \mid \delta_{\theta}\left(\left[\mathrm{e}^{\left.\left.\xi \cdot \mathbf{D}_{g_{12}}\right]^{*} h(\xi ; \cdot)\right) \mid}\right.\right. \\
& \leqq C_{2}\left(1+\left|\xi_{1}\right|\right)^{m_{1}}\left(1+\left|\xi_{2}\right|\right)^{m^{2}}\|\psi\|\|\varphi\|_{m_{3} ; 2}\left\|g_{12}\right\|_{m_{s} ; \infty} .
\end{aligned}
$$

This implies that the function

$$
\xi \rightarrow g_{12}(\xi) \delta_{\theta}\left(\left[\mathrm{e}^{\xi \cdot \mathbf{D}} g_{12}\right]^{*} h(\xi ; \cdot)\right)
$$

is bounded by a Lebesgue integrable function.

Suppose now $\left\{A_{n}: n \geqq 1\right\}$ is any family of Borel rectangles for which there is a Borel set $\Delta \in$ Bor $\left(\mathbf{R}^{2}\right)$ such that the characteristic functions converge pointwise; we write $\Delta_{n} \uparrow \Delta$. Any Borel set $\Delta$ can be obtained this way. It is evident from the Lebesgue dominated convergence theorem, that for any function $F \in \mathscr{S}\left(\mathbf{R}^{2}\right)$, $\lim \delta_{\Delta_{n}}(F) .=\delta_{\Delta}(F)$. It follows that $\Delta_{n} \uparrow d$

$$
\lim _{n}\left\langle Z_{12}\left(\Delta_{n}\right)[b] \varphi, \psi\right\rangle_{N ; 2}=\lim _{n \mathbf{R}^{2}} g_{12}(\xi) \delta_{\Delta_{n}}(h(\xi ; \cdot))=\int_{\mathbf{R}^{2}} g_{12}(\xi) \delta_{\Delta}(h(\xi ; \cdot)) .
$$

Moreover, this last is finite, and

$$
\left|\int_{\mathbf{R}^{2}} g_{12}(\xi) \delta_{\Delta}(h(\xi ;, \cdot))\right|<C\|\psi\|-
$$

for some $C>0$. Taking the supremum over $\psi \in \mathscr{S}(\mathbf{R})$ with $\|\psi\|=1$, we see that $Z_{12}$ is an expectation

\section{Concluding remarks}

In this section we wish to summarize the scheme for quantum mechanical measurements that we have presented, in a schematic and non-technical form. The first remark we feel it is important to make is that our choice of algebra and states is to a great extent determined by the nature of quantum mechanics. An examination of the problems actually treated in quantum theory and attention to the initial development of the subject, shows that its essence lies in the canonical commutation relations. Up to rather moderate technical assumptions, this leads to the space $\mathscr{W}$ of wa, taking direct sums leading immediately to the general case.

The second remark we wish to make is to point out what we did not assume such things as non-repeatibility and instrument distortion. These are results of the mathematical analysis, and must be considered as inherent in the scheme. It seems fair to say that our scheme is essentially operational in origin. We view an instrument, or a measurement, as testing incoming states for some quantum mechanical property and, contingent upon the result observed, emitting an outgoing state. This process should be linear, in accordance with general principles. The emitted state should in fact, be a state on the, algebra, which is why the condition $Q \doteq^{\prime} Z^{\ell}$ occurs. 
Finally, $\sigma$-additivity over the Borel sets seems a modest enough requirement. The other obvious candidate axiom is finite countability. This probably would have consequences for repeatibility, but seems to put an unacceptable constraint on the sorts of measurements which can be performed.

Once our definition of an instrument has been made, it is a mathematical result that we must consider only $(\mathcal{A}, \mathscr{W})$-measures,

$$
\mathscr{M}_{+}(\mathcal{A}, \mathscr{W})=\mathscr{M}_{+}(\mathcal{A}) \cap \mathscr{W}
$$

as the basic material for instruments. This set is seen to replace the set of all orthogonal projections on $\mathfrak{B}$ in the bounded case. It will prove helpful to the intuition to emphasize this, and so we propose to call the $(\mathcal{A}, . \mathscr{W})$-measures questions for the remainder of this section. Evidently it will be useful to analyze the structure of the set of questions and its relation to $\mathcal{A}$. Perhaps an axom scheme based on questions can be devised, generalizing the Mackey scheme to the $(\mathcal{A} ; \mathfrak{W})$ structure.

One way to understand our 'system is to consider the elements of $\mathscr{M}_{+}(\mathcal{A}, \mathscr{W})$ as containing all the definitively answerable quantal questions, and the instruments as the only possible means of answering, them. It is in accord with experience that each instrument answers a unique question whereas each question can be answered in many ways.

Our scheme' determines which operators in the algebra can be measured. First of all we can measure all operators of the form $\int t \mathscr{B}(d t)$, where $\mathscr{A} \in \mathcal{M}_{+}(\mathcal{A}, \mathscr{W})$ is a question. These constitute a rather special class of operators: we shall come back to this point below. The most general symmetric observables which can be measured aire those $b \in \mathcal{A}_{\mathrm{h}}$ which admit of a spectral decomposition by at least one $\mathcal{A}$-measure $\mathscr{B}$; which has more information than at least one question $\mathscr{C} \in \mathscr{M}_{+}(\mathscr{A}, \mathscr{W})$. That is, $\mathscr{B}>\mathscr{C}$. Then $\mathscr{C}$ is a question we can answer which will tell us something about $b$. The most we can known about $b$ is contained in all the questions $\mathscr{C}$ satisfying $\mathscr{C}<\mathscr{B}$ as we run through all the spectral decompositions of $b$. If $b$ is essentially self-adjoint, there is only one such decomposition.

In the usual description of quantum measurement theory, a measurement has a dual function. Firstly it determines eigenvalues, more generally spectral values; secondly it prepares states by virtue of the postilate of collapse into eigenstates. There are two sorts of instrument distortion therefore: imperfect reading, of the spectral values, and imperfect filtration of eigenstates. These latter possibility is related to non-repeatibility, of course. An ideal instrument is one which admits of neither sort of distortion. It is a mathematical result in our' scheme that such instruments do not exist, except for operators in $\mathcal{A}_{\mathrm{h}}$ whose spectrum consists only of isolated eigenvalues of finite multiplicity. For operators defined by spectral syntheses from questions, these seems to be no bar to instruments which do not distort the spectral values. Nonetheless, as there is no repeatibility, there is always spectral measure distortion present. This can be seen from eq. (3.21) by acting with $Q(\Delta)$ twice. The compact operators are such that there distortions, around an eigenprojection can be made small enough not to suffer interference from the neighbouring eigen-projections. Although not to be taken too seriously, a mental image might be of a beam incident on a slit: The slit edges must not be sharply defined, but must be $\mathscr{S}_{\text {;-class }}$ so as not to chop off the beam too sharply. An observer reads the eigenvalues of the beam as it passes through, but the observer is, typically, near sighted. For our special observables, the eigenvalues are written in sufficiently large type that there need be no distortion. A's if this were not strange enough, the whole slit apparatus has an uncontrollable tremor, causing imperfect filtration of spectral projections.

Another point. worth emphasizing is that the above considerations hold for one observable alone; the uncertainty principle interference effects arè not being con- 
sidered. If one demands "states in, states out" and $\sigma$-additivity, it is a mathematical consequence that a perfect measurement is impossible. Considering $Q$ measurenents again, one can only measure observables obtained from questions and satisfying $Q>b$. For example, one can measure $F *_{-\varkappa}(Q)$ for all $F(x)=|f(x)|^{2}$; all $f \in \mathscr{I}(\mathbf{R})$. By judicious choice of $f$ one can get close to $Q$ in some suitable sense, but $Q$ itself cannot actually be measure. The function $f$ both distorts the spectral readings of $Q$ and the spectral projection filtration. Were one to wish to measure $F * \chi(Q)$ rather than $Q$, spectral projection distortion would still occur in the sense of non-repeatibility.

\section{REEERENCES}

[1] Choquet, G.: Lectures on Analysis, Vol. I. New York: Benjamin 1969.

[2] Davies, E. B.: On the Repeated Measurement of Continuous Observables in Quantum , Mechanics. J. Funct. Anal. 6.(1970), 318-346.

[3] Davies, E. B.: Quantum Theory of Open Systems. London-New York-San Francisco: Academic Press 1976.

[4] Davies, E. B., and J. T. Lewis: An Operational Approach to Quantum Probability. Comm. Math. Phys. 17 (1970), 239-260.

[5] Drestel, J., land J. J. UHL: Vector. Measures. Providence: Amer. Math. Soc. 1977.

[6] Gez'Fand, I. M., and N. YA. Vilenkin: Generalized Functions IV. New York-London: Academic Press 1964.

[7] Holevo, A. S.: The Analogue of Statistical Decision Theory in Noncommutative Probability Theory. Trans. Moscow Math. Soc. 26 (1972), 113-149.

[8] Holevo, A. S.: Statistical Decision Theory for Quantum,Systems. J. Mult. Anal. (1975), 337-394.

[9] Hoxzrker, W.: On the Space-Time Behaviour of Schrödinger Wavefunctions. J. Math. Phys. 7 (1966), 300.

[10] JaRChow, H.: Locally Convex Spaces. Stuttgart: Teubner-Verlag 1981.

[11] Jrizzak, J.-P.: Simple Facts about Algebras of Unbounded Operators. J. Funct. Anal. 21 (1976), $469-482$.

[12] Kriștensen, P., Mejibo, L., and E. Thue Poursen: Tempered Distributions in Iñfinitely Many Dimensions I. Comm. Math. Phys. 1 (1965); 175-214.

[13] KötнE, G.: Topological Vector Spaces: I. Berlin-Heidelberg-New York: SpringerVerlag 1969.

[14] Kӧтне, G.: Topological Vector Spaces II. Berlin-Heidelberg-New York: SpringerVerlag 1979.

[15] LasSner, G.: Topological Algebras of Operators. Rep. Math. Phys. 3 (1972), 279-293.

[16] Lassner, G.: Mathematische Beschreibung von Observablen-Zustandssystemen. Wiss. Z. Karl-Marx-Univ. Leipzig, Math.-Naturwiss. R. 2 (1973), 103-138.

[17] LaSSNer, G.: Topological Algebras and their Applications in Quantum Statistics. Inst. Phys. Th., Univ: Catholique de Louvain: Lecture Notes UCL-IPT-80-09 (1980).

[18] Lassner, G., and G. A. LAssner: On the Continuity of Entropy. Rep. Math. Phys. 15 (1979), $41-46$.

[19] Lassner, G., and W. Thmmermann: Normal States on Algebras of Unbounded Operators. Rep. Math. Phys. 3 (1972), 295-305.

[20] Lassner, G., and W. Trmmermann: Classification of Domains of Closed Operators. Rep. Math. Phys. 9 (1976), 157-170.

[21] Lassner, G., and A. Umlimane: On Op*-Algebras of Unbounded Operators. Proc. Steklov Inst. Math., AMS transl. 1978, $171 \div 176$.

[22] Lassner, G. A.: Operator Symbols in the Description of Observable-State Systems. Joint: Inst. Nuclear Res. (Dubna). Preprint E2-11270 (1978).

[23] Namaḱ, M. A.: Normed Rings. Groningen: Noordhoff 1964.

[24] Neumans, J. vos: Mathematische Grundlagen der Quantenmechanik. Berlin: Springer Vering 1932. 
[25] Parthasarathy, K. R.: Introduction to Probability and Measure. London: MacMillan 1977.

[26] Piersch, A.: Nuclear Locally Convex Spaces. Berlin-Heidelberg-New York: SpringerVerlag 1972.

[27] Powers, R. T.: Self-Adjoint Algebras of Unbounded Operators. Comm. Math. Phys. 21 (1971), 85-124.

[28] Potnam, C. R.: Commutation Properties of Hilbert Space Operators and Related Topics. Berlin-Heidelberg-New York: Springèr-Verlag 1967.

[29] Reen, M., and B. Simon: Methods of Modern Mathematical Physics I: Functional Analysis. New York-London: Academic Press 1972.

[30] Riesz, F;, and B. Sz-NAGY: Functional Analysis, Appendix. New York: F. Ungar 1960.

[31] Roвerts, J. E.: The Dirac bra and ket formalism. J. Math. Phys. 7 (1966), 1097-1104.

[32] Roberts, J. E.: Rigged Hilbert Spaces in Quantum Mechanics. Comm. Math. Phys. 3 (1966), 98-119.

[33] Rodis, W.: Principles of Mathematical Analysis, 2nd Ed. Tokyo: McGraw-Hill-Kōgakusha 1964.

[34] Rodrs, W.: Real and Complex Analysis. New Delhi: Tata, for MeGraw-Hill, New York 1974.

[35] Schaffer, H. H.: Topological Vector Spaces. Berlin-Heidelberg-New York: SpringerVerlag 1971.

[36] ScmïugeN, K.: The Order. Structure of Topological *-Algebras of Unbounded Operators I. Rep. Math. Phys. 7 (1975), 215-227.

[37] Schmüngex, K.: On Trace Representation of Linear Functionals on Unbounded Operator Algebras. Comm. Math. Phys. 63 (1978), 113-130.

[38] ScmüUden, K.: On 'Topologization of Unbounded Operator Algebras. Rep. Math. Phys. 17 (1980), 359-371.

[39] Sherman, T.: Positive Linear Functionals on *-Algebras of Unbounded Operators. J. Math. Anal. Appl. 22 (1968), 285-318.

[40] Simon, B.: Distributions and their Hermite Expansions. J. Math. Phys. 12 (1971), $140-148$.

[41] Sotelo-Campos, J.: An Application of Operator *-Algebras to the Quantum Theory of Measurements. Thesis. Milton Keynes (England): The Open University 1983.

[42]. Thomas, E.: L'integration par rapport à une measure de Radom vectorielle. Ann. Inst. Fourier (Grenoble) 20 (1970) 2, 55-191.

[43] Tномаs; E.: The Lebesgue-Nikodym Theorem for Vector Valued Measures. Mem. Amer. Math. Soc. 139 (1974).

[44] Treves, F.: Topological Vector Spaces, Distributions and Kernels. New York-London: Academic Press 1967.

[45] Woronowicz, S. L.: The Quantum Problem of Moments. Rep. Math. Phys. 1 (1970); $175-183$.

[46] Wright, J. D. M.:' Products of Positive Vector Measures. Quart. J. Math. (Oxford) (2) 24 (1973), 189-206.

[47] Yosida, K.: Functional Analysis. Berlin-Heidelberg-New York : Springer-Verlag 1980.

- Manuskripteingang: 23. 05. 1984.

\section{VERFASSER:}

Prof. Dr. Daniel A. Dubin and Dr. J. Sotelo-Caypos

Ficulty of Mathematies, The Open University

England - Milton Keynes, MK 7 6AA, Walton Hall 